\title{
Mechanistic Modelling - a BOLD response to the fMRI information loss problem
}

\author{
Karin Lundengård
}

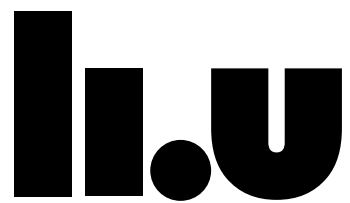

LINKÖPING UNIVERSITY

Linköping University medical dissertations, No. 1591

Department of Medical and Health Sciences (IMH)

Centre for Medical Image Science and Visualization (CMIV)

Linköping University 
Cover page: Classic BOLD response in the colors of activation.

Date of defense: 2017-11-30.

ISBN: 978-91-7685-441-9, ISSN: 0345-0082

Printed by LiU-Tryck, Linköping 2017 
To everyone who let me take a look at their brain, particularly the pilots; the test participants get all the credit, even though you are the ones spending all that extra time in the scanner to sort out the messes we make. Without you, none of our experiments would be running at all. 


\section{Populärvetenskaplig sammanfattning}

När vi studerar hur människans hjärna fungerar är det viktigt att använda en teknik som är säker för försökspersonerna och samtidigt ger relevant information om hjärnan. Funktionell Magnetresonanstomografi (fMRI) kan snabbt avbilda hela hjärnan om och om igen på samma person. Från bildserien kan man använda fMRI-signalen för att mäta lokala förändringar i syrehalt i olika delar av hjärnan. Syrehalten ändras på grund av att nervcellerna använder mer syre när de arbetar mer. Men för att cellerna inte ska få brist på syre och glukos vidgas blodkärlen för att skicka dit mer blod, och det ändrar också fMRI-signalen. Även om nerverna bara tar några millisekunder på sig att skicka signaler så kan syreförändringen vi mäter pågå upp till 20 sekunder. Den stora tidsskillnaden gör det svårt att lista ut hur nervaktiviteten ser ut genom att bara titta på fMRI-signalen. Hur hjärncellerna påverkar blodkärlen är vi inte helt säkra på, men det finns flera olika hypoteser.

Den hypotes som vi har undersökt beskriver hur när nerverna i hjärnan startar en signalkedja som får andra typer av hjärnceller att skicka ut signalsubstanser som i sin tur styr hur blodkärlen vidgar sig eller drar ihop sig när nervaktiviteten ökar. I det här projektet översätter vi hypotesen till matematiska ekvationer i en datormodell som efterliknar kommunikationen mellan hjärncellerna och blodkärlen. Först en datormodell. Sedan visar jag att modellen kan simulera data som vi har tränat den på. Den kan även korrekt förutsäga hur hjärnaktiviteten borde se ut i nya experiment där stimulit varierar på ett sätt som modellen inte har sett innan. Efter det visar vi att modellen kan se skillnad på när det finns hjärnaktivitet i signalen och när det bara finns brus, samt bedöma hur starkt stimulit är. Detta gör vi genom att använda oss av en inre egenskap i modellen, nämligen hur mycket hjärncellerna säger till blodkärlen att vidga sig, istället för att bara titta på fMRI-signalen. I dessa tester använder vi simulerade data för att vara säker på vad det rätta svaret är, eftersom vi inte alltid vet hur aktiva hjärncellerna är i verkligheten.

Modellen kan också simulera inhibering, vilket innebär att något område i hjärnan förhindras från att aktiveras. Inhibering är en viktig egenskap eftersom den reglerar de olika nätverken av hjärnområden så att de inte stör ut varandra när de utför olika uppgifter. Den sista egenskapen vi undersöker med modellen är att simulera vad som händer när man tillför det lugnande ämnet diazepam. Det finns ett område i hjärnan som blir inhiberat när man får diazepam. Med modellen kan vi visa att det är för att en viss receptor blir känsligare för ämnen som dämpar hjärnaktivitet när man har fått diazepam. Förhoppningsvis kommer vår modell och metoderna som vi har utvecklat i framtiden att ge oss djupare förståelse av hur hjärnan fungerar, samt hitta biomarkörer som kan diagnosticera störningar och sjukdomar i hjärnan som vi idag saknar möjlighet att testa för. 


\section{Abstract}

Functional Magnetic Resonance Imaging (fMRI) is a common technique for imaging brain activity in humans. However, the fMRI signal stems from local changes in oxygen level rather than from neuronal excitation. The change in oxygen level is referred to as the Blood Oxygen Level Dependent (BOLD) response, and is connected to neuronal excitation and the BOLD response are connected by the neurovascular coupling. The neurons affect the oxygen metabolism, blood volume and blood flow, and this in turn controls the shape of the BOLD response. This interplay is complex, and therefore fMRI analysis often relies on models. However, none of the previously existing models are based on the intracellular mechanisms of the neurovascular coupling. Systems biology is a relatively new field where mechanistic models are used to integrate data from many different parts of a system in order to holistically analyze and predict system properties. This thesis presents a new framework for analysis of fMRI data, based on mechanistic modelling of the neurovascular coupling, using systems biology methods.

Paper I presents the development of the first intracellular signaling model of the neurovascular coupling. Using models, a feed-forward and a feedback hypothesis are tested against each other. The resulting model can mechanistically explain both the initial dip, the main response and the post-peak undershoot of the BOLD response. It is also fitted to estimation data from the visual cortex and validated against variations in frequency and intensity of the stimulus. In $\mathrm{Pa}$ per II, I present a framework for separating activity from noise by investigating the influence of the astrocytes on the blood vessels via release of vasoactive substances, using observability analysis. This new method can recognize activity in both measured and simulated data, and separate differences in stimulus strength in simulated data. Paper III investigates the effects of the positive allosteric GABA modulator diazepam on working memory in healthy adults. Both positive and negative BOLD was measured during a working memory task, and activation in the cingulate cortex was negatively correlated to the plasma concentration of diazepam. In this area, the BOLD response had decreased below baseline in test subjects with $>0.01 \mathrm{mg} / \mathrm{L}$ diazepam in the blood. Paper IV expands the model presented in Paper I with a GABA mechanism so that it can describe neuronal inhibition and the negative BOLD response. Sensitization of the GABA receptors by diazepam was added, which enabled the model to explain how changes to the BOLD response described in Paper III could occur without a change in the balance between the GABA and glutamate concentrations.

The framework presented herein may serve as the basis for a new method for identification of both brain activity and useful potential biomarkers for brain diseases and disorders, which will bring us a deeper understanding of the functioning of the human brain. 
Supervisor:

\section{Maria Engström}

Professor

Department of Medical and Health Sciences

Centre for Medical Image Science and Visualization (CMIV)

Linköping University

Co-supervisors:

\section{Gunnar Cedersund}

Associate Professor

Department of Biomedical Engineering

Department of Clinical and Experimental Medicine

Linköping University

\section{Fredrik Elinder}

Professor

Department of Clinical and Experimental Medicine

Linköping University

\section{Susanna Walter}

MD, Associate Professor

Department of Clinical and Experimental Medicine

Centre for Medical Image Science and Visualization (CMIV)

Linköping University

Opponent:

\section{Kâmil Uludağ}

Associate Professor

Faculty of Psychology and Neuroscience

Maastrich University 


\section{Summary of Papers}

\section{Mechanistic mathematical modeling tests hypotheses of the neurovascular coupling in fMRI}

Karin Lundengård, Gunnar Cedersund, Sebastian Sten, Felix Leong, Alexander Smedberg, Fredrik Elinder, Maria Engström. PLoS Comput. Biol, 2016

A set of new, mechanistic models of the neurovascular coupling and the positive BOLD response are presented. The metabolic hypothesis and the neurotransmitter hypothesis are tested against each other. The resulting model can mechanistically explain the initial dip, the post-stimullus peak and the post-peak undershoot of the BOLD response, as well as describe estimation data and predict validation data.

\section{Biomarkers in fMRI based on biological mechanisms}

Karin Lundengård, Sebastian Sten, Maria Engström, Fredrik Elinder, Gunnar Cedersund. Manuscript

A new framework for analyzing brain activity based on observability of model properties is presented. It can separate activity from noise in measured data, as well as levels of activity from different stimulus strength in simulated data by measuring the glial influence on the blood vessels.

\section{Positive allosteric modulator of GABA lowers BOLD responses in the cin- gulate cortex}

Susanna Walter, Mikael Forsgren, Karin Lundengård, Rozalyn Simon, Maritha Torkildsen Nilsson, Birgitta Söderfeldt, Peter Lundberg, Maria Engström. PLoS One, 2016

The effects of the positive allosteric GABA modulator diazepam on working memory activation in the brain is investigated. Data showed both positive and negative activation during the task, and activation in cingulate cortex was negatively correlated to the plasma concentration of diazepam. The BOLD response changes from positive to negative for the test subjects with $>0.01 \mathrm{mg} / \mathrm{L}$ diazepam in the blood,

IV. Neural inhibition can explain negative BOLD responses: A mechanistic modelling and fMRI study

Sebastian Sten, Karin Lundengård, Suzanne T Witt, Gunnar Cedersund, Fredrik Elinder, Maria Engström. Neuroimage, 2017

The model from Paper I is expanded with a GABA signaling mechanism to describe neuronal inhibition and the negative BOLD response. The sensitizing effect of diazepam on GABA receptors was modelled, and the model showed that a decrease in the BOLD response could be explained without a change in the balance between the GABA and glutamate concentrations. 


\section{Abbreviations}

$\begin{array}{ll}\text { AA } & \text { Arachidonic Acid } \\ \text { AMPA } & \alpha \text {-amino-3-hydroxy-5-methyl-4-isoxazolepropionic Acid } \\ \text { ASL } & \text { Arterial Spin Labeling } \\ \text { ATP } & \text { Adenosine Triphosphate } \\ \text { BOLD } & \text { Blood Oxygen Level Dependent (response) } \\ \text { CBF } & \text { Cerebral Blood Flow } \\ \text { CBV } & \text { Cerebral Blood Volume } \\ \text { CEN } & \text { Central Executive Network } \\ \text { dHb } & \text { deoxy-Hemoglobin } \\ \text { DMN } & \text { Default Model Network } \\ \text { EEG } & \text { Electroencephalography } \\ \text { EET } & \text { Epoxyeicosatrienoic Acid } \\ \text { fMRI } & \text { functional Magnetic Resonance Imaging } \\ \text { GABA } & \gamma \text {-Aminobutyric Acid } \\ \text { GLM } & \text { General Linear Model } \\ \text { Hb } & \text { Hemoglobin } \\ \text { ISI } & \text { Inter-stimulus Interval } \\ \text { KLS } & \text { Kleine Levin Syndrome } \\ \text { NMDA } & \text { N-methyl-D-aspartate (receptor) } \\ \text { ODE } & \text { Ordinary Differential Equation } \\ \text { oHb } & \text { oxy-Hemoglobin } \\ \text { PDE } & \text { Partial Differential Equation } \\ \text { PET } & \text { Positron Emission Tomography } \\ \text { PPL } & \text { Prediction Profile Likelihood } \\ \text { SE } & \text { Standard Error } \\ \text { SN } & \text { Salience Network } \\ \text { VDCC } & \text { Voltage-Dependent Calcium Channels } \\ \text { 20-HETE } & \text { 20-Hydroxyeicosatetraenoic Acid } \\ & \end{array}$




\section{Contents}

1 Introduction $\quad 3$

1.1 A Short History of Models in Brain Science . . . . . . . . . . . . 4

1.2 A Short History of fMRI . . . . . . . . . . . 5

2 Imaging the Brain With fMRI $\quad 7$

2.1 What Does the fMRI Scanner Measure? . . . . . . . . . . . 7

2.2 The Blood Oxygen Level Dependent Response in fMRI . . . . . . 8

2.3 The Neurovascular Coupling . . . . . . . . . . . . . . . . . 11

2.4 The Importance of Inhibition . . . . . . . . . . . . . . 15

2.5 Pharmacological Modulation of GABA . . . . . . . . . . 16

2.6 The Structure of fMRI-Images . . . . . . . . . . . . . . . 16

2.7 fMRI Noise . . . . . . . . . . . . . . . . . . . . . . 18

2.8 Preprocessing of fMRI Images . . . . . . . . . . . . . . . . . . 19

2.9 fMRI Experiment Design . . . . . . . . . . . . . . . . 20

2.10 Analysis of fMRI Data . . . . . . . . . . . . . . 23

2.11 The fMRI Information Loss Problem . . . . . . . . . . . . 24

3 Systems Biology 29

3.1 System Properties . . . . . . . . . . . . . . . . . . . . . . . . 29

3.2 What Defines Systems Biology? . . . . . . . . . . . . . . . . 29

3.3 Modelling Biology With Mechanistic Models . . . . . . . . . . . 30

3.4 Ordinary Differential Equations and Model Formulation . . . . . 33

3.5 Model Minimization . . . . . . . . . . . . . . . . . . 36

3.6 Model Uncertainty and Analysis of Identifiability and Observability 37

$\begin{array}{lll}4 & \text { Aims } & 43\end{array}$

5 Results $\quad 45$

5.1 Aim 1: Model development . . . . . . . . . . . . . . . 45

5.2 Aim 2: Explain the Positive and the Negative BOLD Response Through Activation and Inhibition . . . . . . . . . . 46 
5.3 Aim 3: Potential Biomarkers . . . . . . . . . . . . . . 48

5.4 Aim 4: Pharmaceutical Modulation of GABA . . . . . . . 50

6 Discussion 55

6.1 Systems biology for hypothesis testing . . . . . . . . . . 55

6.2 Potential applications in network studies . . . . . . . . . 56

6.3 Biomarkers and personalized medicine . . . . . . . . . 56

$\begin{array}{ll}\text { Conclusion } & 58\end{array}$

Acknowledgements 
"With its billions of interconnected neurons, whose interactions change from millisecond to millisecond, the human brain is an archetypal complex system." Miguel Nicolelis 


\section{Chapter 1}

\section{Introduction}

The brain is where our personality, memories and all our knowledge is stored. Put simply, it contains information about everything that makes us who we are and it is exactly this which makes it so intriguing to study. The brain performs many functions simultaneously, several of which arise not from isolated areas but from interactions between different areas of the brain, forming networks which can in turn interact with each other [1][2]. The brain is therefore a complex system, making it hard to study. In this thesis, I bring together two powerful but young techniques, namely functional magnetic resonance imaging (fMRI) and systems biology, in order to build a new method for extracting information from fMRI images for the study of brain function.

The development of fMRI took the neuroscience field with storm and despite the fact that the first fMRI study was published as late as 1992 [3], it is now a well established technique in brain science as well as in the clinic. It is also a fairly complex technique where both the generation, acquisition and analysis of the data include many steps before a result is achieved. Computers are essential tools in all steps of this process and with their superior processing power they open the way for increasingly complex analyzing methods. Systems biology is a research field focused on using computerized models to manage large datasets in order to gain a holistic view of the properties of biological systems [4]. Using the methods from systems biology as a framework it might be possible to extract information about the functioning of the brain which has so far been hidden in the fMRI signal.

But before we go into the technical details, let us start at the beginning with a short historical overview of brain models and fMRI. 


\subsection{A Short History of Models in Brain Science}

When understanding a system as complex as the brain, it is useful to employ simplifications and metaphors to build explanations of the structure and function of the system. In the sciences, such simplifications and metaphors are called "models." Humans have employed models for a long time. In fact the oldest known model for the function of the brain comes from descriptions of Egyptian mummification rites, where the brain is described as a gland whose only function is to produce mucus. In a slightly younger document from 1700 BC, written by Egyptian battle surgeons and copied in the Edwin Smith Papyrus [5], the first text connecting brain damage to symptoms such as aphasia and convulsions can be found. Throughout history, many civilizations have had their own hypotheses about the function of the brain, and over the centuries the anatomy and function of the brain and brainstem have been described in greater and greater detail. In 1816, Mary Shelley was inspired by the works of Galvani and Volta on the role of electricity in neurons and muscles to write "Frankenstein; or the Modern Prometheus" [6]. However, the concept of "neuron" itself did not emerge until 1891, as Ramon y Cajals work on portraying the anatomy of brain tissue resulted in the neuron doctrine, for which he shared the Nobel Price in Physiology or Medicine with Golgi [7]. Ironically enough, Golgi was the one who invented the staining techniques used by Ramon y Cajal, but in his Nobel lecture he promoted a competing hypothesis about neuronal function [8]. During the same era, Broca and Brodmann each studied the structure of the brain and discovered that different centra in the brain had different functions. Brodmanns work resulted in histological maps which are still used today to indicate the location of specific structures in the brain. It was not until the 20th century that neuroscience became its own area of research, but since then a plethora of new technical equipment and new methods for studying the brain have sprung forth. The first mathematical model of neuronal function, and indeed the first model in what would later become the field of systems biology, was Hodgkins and Huxleys mechanistic model of the generation of action potentials in neurons [9]. The Hodgkin and Huxley model is by modern standards simple, but nevertheless both useful and elegant. The development of computers have since opened up new possibilities in the development of computing methods, measuring techniques and data storage. This in turn have enabled a rapid proliferation and development of computerized brain models and analysis methods for all sorts of brain data. 


\subsection{A Short History of fMRI}

The history of fMRI begins even before the MRI scanner was invented, with the discovery made by Roy et al. in the late eighteenhundreds that cerebral blood flow was connected to neuronal activity [10][11].In 1936 the next piece of information paving the way for fMRI came from Paulin and Coryell and their discovery that the magnetic property of hemoglobin is dependent on whether or not it is oxygenated [12]. Forty years later, in 1977, the first MRI scan of a human took place [13], but it was not until 1990, Ogawa et al. put together the two fundamental pieces of neuronal blood flow control and the effect of hemoglobin oxygenation on the magnetic field of the scanner. In an elegant series of experiments, using electroencephalography (EEG) to confirm the correlation, they showed that MRI could be used to measure brain activity with blood as an internal contrast [14]. The fillowing year when the first fMRI data collected in humans was presented, by Kwong et al. who showed activity in the visual cortex of a healthy volunteer [3] and by Belliveau et al. who used gadalinium to show changes of the CBV during brain activity [15]. Before this, positron emission tomography (PET) and EEG had been the predominant techniques for investigating brain activity in humans. However, with its noninvasive nature, superior spatial resolution to both previous techniques and a far better temporal resolution than PET, fMRI quickly became the technique of choice in many neuroscience studies. From no publications at all before 1990, there were more than 2000 publications of studies performing fMRI on humans in the year 2012 alone [16]. But what type of information can we get from fMRI? Let us take closer look at this popular technique in the next chapter. 
"It appears rather gruesome:

Wrinkled like a walnut, and with the consistency of mushroom." 


\section{Chapter 2}

\section{Imaging the Brain With fMRI}

\subsection{What Does the fMRI Scanner Measure?}

Functional magnetic resonance imaging (fMRI) is the technique of measuring brain activity with a magnetic resonance imaging (MRI) scanner. This type of scanner uses a strong magnetic field and radio frequency pulses to measure properties of the nuclear magnetic spin of the protons in the body. Since these properties are affected by what type of atoms and molecules the protons are in, it is possible to calculate what kind of tissue the signal is coming from and this information is then used to build images of what the body looks like on the inside. As the body consists mostly of water, which contains many protons in the form of hydrogen, the bulk of the MRI signal comes from hydrogen protons. "Functional" in the context of fMRI means that we are interested not only in the anatomy of the person we are scanning, but also of how something changes in their body, e.g. the brain activity. In fact, measurements specifically of brain activity is now what most people refer to when they say "fMRI." When measuring brain activity with fMRI, the blood acts as an internal contrast. This means that the blood itself can enhance the fMRI signal, without any foreign substances being added to the body. The signal enhancement comes from the hemoglobin $(\mathrm{Hb})$ present in the blood. Hemoglobin transports oxygen throughout the body by binding oxygen when the blood is in an area with a high concentration of oxygen and then release it when the blood reaches tissue with a low concentration of oxygen. Hemoglobin bound to oxygen is called oxy-hemoglobin $(\mathrm{oHb})$ and hemoglobin which has already released its oxygen is called deoxy-hemoglobin $(\mathrm{dHb})$. oHb is diamagnetic and does not effect the fMRI signal, but $\mathrm{dHb}$ is paramagnetic and causes the fMRI signal to decrease. It is possible to localize brain activity by measuring signal changes over time caused by changes in the levels of $\mathrm{dHb}$, which are connected to the neuronal activity. 
We will get back to the more technical aspects of fMRI measurements and analysis in section 2.6 and subsequent sections, but first we will take a look at the biological mechanisms behind the measurements.

\subsection{The Blood Oxygen Level Dependent Response in fMRI}

As already stated in the previous section, fMRI depends on changes in oxygenation over time in the brain. Ogawa, whose group was the first to use this change to measure brain activity, named it the Blood Oxygen Level Dependent response, or the BOLD response [14]. It is also known as the hemodynamic response. The BOLD response has a very distinct shape. A classic BOLD response to a short stimulus can be seen in Figure 2.1A. The BOLD response is closely correlated to neuronal excitiation and local field potentials, and we will go through the biological mechanisms generating the BOLD response in section 2.3. Here, we start by examining the shape of the BOLD response. The first thing that happens in the BOLD response is a slight signal drop, called the initial dip. Some argue that this initial dip is the best measurement of the true neuronal activity, since it is hypothesized that it is caused by an increase in oxygen metabolism [17], which is a direct response to increased neuronal excitation. The initial dip is a very small signal change [18] and can not be detected on all scanners or in all tasks [18][19]. The relevancy, and even existence of the initial dip is debated, and some studies argue that it might be due to artifacts caused by the measuring techniques used, and not connected to the neuronal workload [20]. Therefore, the most common part of the BOLD response to focus on is the next part of the response, which is the poststimulus peak. The post-stimulus peak is caused by a vascular response where blood flow (CBF) and blood volume (CBV) in the active area is increased, which increases the fMRI signal. The post-stimulus peak represents a bigger change in the signal (about $2-5 \%$ of total signal) than the initial dip, and is more easily recognized by most types of fMRI analysis than the initial dip. The increase in signal is caused by changes in CBF and CBV, which affect the signal by increasing the oxygenation level. The post-stimulus peak usually appears $5-15$ seconds after the stimulus, and the long time lag is attributed to the relative slowness of the muscles in the blood vessel walls. The fact that the peak of the BOLD response is very slow compared to the underlying neuronal processes must be taken into account when fMRI experiments are constructed (more on that in section 2.9). If there is no further stimulus, the signal falls back to baseline again after the peak, usually with an oscillating behavior called the post-peak undershoot. It is most common to attribute the post-peak undershoot to either the biomechanics of the blood and 

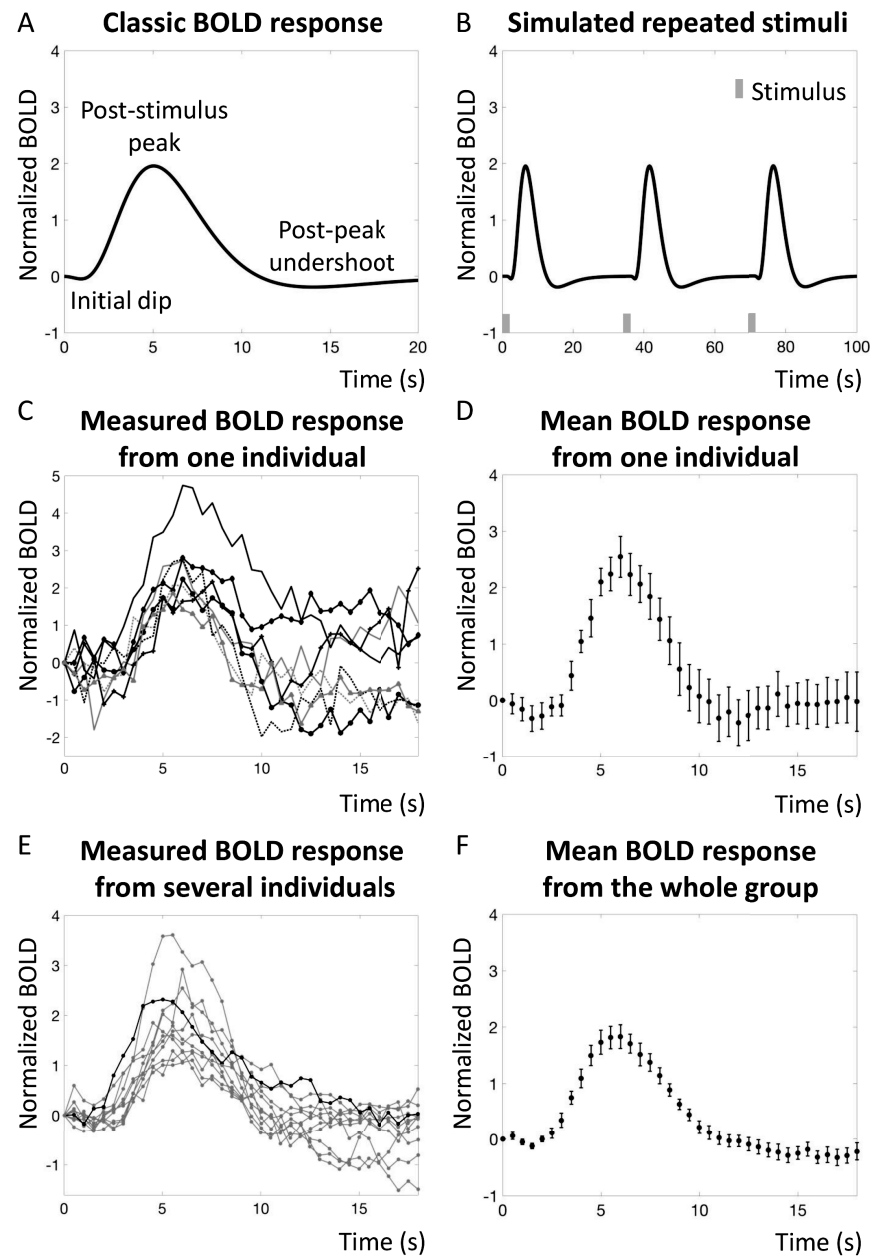

Figure 2.1: The positive BOLD response. A. The classical representation of the BOLD response as it is often described in the litterature. B. Model simulation of the positive BOLD response to the same repeated stimulus. A new, but identical, BOLD response appears after each stimulus. C-F. Data collected in the visual cortex in response to a short visual stimulus $(0.5 \mathrm{~s})$, with at least $15 \mathrm{~s}$ before the next stimulus. All errorbars represent mean and SE. C. Measured BOLD responses to the same repeated stimulus in the same area in the same individual can still have a very different appearance. D. When the data from one individual is averaged, the shape of the classical BOLD response is more discernible. E. Mean BOLD responses from different individuals. F. Group mean of the BOLD response to a short stimulus. 
the blood vessels [21][22][23][24] or a slow metabolism of oxygen [17], but it has also been suggested that the post-stimulus undershoot is connected to poststimulus neuronal activity [25].

The total time for a BOLD response to a short stimulus is approximately 1520 seconds. The long lag of the fMRI signal compared to neuronal excitation, combined with the change in the shape of the signal caused by factors other than the neuronal activity (such as the $\mathrm{CBF}$ and $\mathrm{CBV}$ ) makes it hard to infer the true neuronal activity from fMRI measurements. However, since the BOLD response is correlated to neuronal spiking and local field potentials [26][27][28], identification of the BOLD response is indeed useful to locate areas of increased activity in the brain.

The ideal measuring situation of the BOLD response is the one simulated in Figure 2.1B, where each identical stimulus is followed by an identical BOLD response. This is also the way that the models in the papers presented herein have simulated the BOLD response. Of course this ideal situation is only ever observed in deterministic model simulations. Figure 2.1C shows several BOLD responses measured in the same area of the brain of one individual, for the same stimulus during the same experimental session. Despite that, the all BOLD responses have a different shape. The shape of every individual BOLD response may depend on what type of stimulus preceded the current one, how long time has passed since the previous stimulus, what part of the brain is measured, what other processes are going on at the same time in other parts of the brain, and several other factors, most of which are unknown to the researcher. In Figure 2.1D, the mean and standard error (SE) of all the individual responses have been calculated, and now the classical shape of the BOLD response is more easily discernible. But even though the mean curve follows the shape of the classic BOLD response, the mean curve from different individuals can still look very different, as shown in Figure 2.1E. Figure $2.1 \mathrm{~F}$ shows the mean curve and $\mathrm{SE}$ of the mean curves of the group from $\mathrm{E}$, and this is the type of data that was used when fitting and testing the model presented in Paper I. All graphs showing the BOLD response in this thesis starts at zero because the signal change has been normalized to show only the percent signal change. However, it is important to note that the BOLD response is a change from the baseline signal, and that the baseline fMRI signal is never zero, since the brain is always performing multiple functions simultaneously.

It should be noted here that there are fMRI techniques which measure other things than the BOLD response (such as e.g. Arterial Spin Labeling, ASL), but since BOLD-fMRI is the most common type of fMRI, it is also the technique most often referred to when the expression fMRI is used.

Several times already I have stated that the BOLD response is correlated with neuronal activity. However, correlation does not necessarily imply causality, so what are the mechanisms connecting the neuronal activity and the BOLD response 
measured in the fMRI signal?

\subsection{The Neurovascular Coupling}

The neurovascular coupling is the chain of mechanisms connecting the neuronal activity with the actions of the blood vessels, and thus it is the underlying mechanisms behind the BOLD response. The neurovascular coupling is what makes it possible for us to measure brain activity with fMRI at all. There are several hypotheses focusing on different aspects of the neurovascular coupling, proposing different mechanisms to explain the neurovascular coupling, and differing in how relevant each of these mechanisms are considered to be.

\section{Hypotheses of the Neurovascular Coupling}

One of the oldest hypotheses of the main mechanism behind the BOLD response is the metabolic control of the blood flow. In 1986 [29] Fox et al. hypothesized that an increased workload of the neurons leads to an elevated metabolism of glucose, which in turn causes CBF to increase in the activated area of the brain, in order to replenish the glucose supply. The metabolism of oxygen does not increase as much as the glucose metabolism, possibly because of a switch from aerobic to anaerobic metabolism (known as an uncoupling mechanism) during increased activity, and the excess oxygen in the blood cause the peak of the BOLD response.

A more modern and widely used hypothesis is the balloon model presented by Buxton [30] and Friston [31]. In the Balloon model, increased CBF builds pressure which makes the blood vessels expand, increasing the CBV, and the accumulation of more oxygen rich blood increase the fMRI signal. The original balloon model focuses only on mechanisms in the blood vessels and oxygen metabolism, and omits intracellular mechanisms.

In the experimental field, interest in the intracellular mechanisms of the neurotransmitter feed-forward hypothesis illustrated in Figure 2.2A dominates. The modelling framework presented in this thesis rests on the neurotransmitter feedforward hypothesis, and we shall therefore focus a bit more on the details of this hypothesis. The model representation of this system presented and tested in Paper IV is illustrated in section 5.1, to show the corresponding parts of the biological system and the model. The neurotransmitter feed-forward hypothesis rests on data which are excellently reviewed in [32][33][34]. In short, the presence of neurotransmitters in the synaptic cleft generates an action potential in the post-synaptic neuron, which triggers the release of ATP outside of the neuron activates $\mathrm{P}_{2} \mathrm{X}_{1}$ receptors in adjacent astrocytes resulting in $\mathrm{Ca}^{2+}$ influx into the astrocyte. The inflow of $\mathrm{Ca}^{2+}$ activates phospholipase D2 (PLD2) which, after some enzymatic 


\section{Neurovascular signalling pathway}

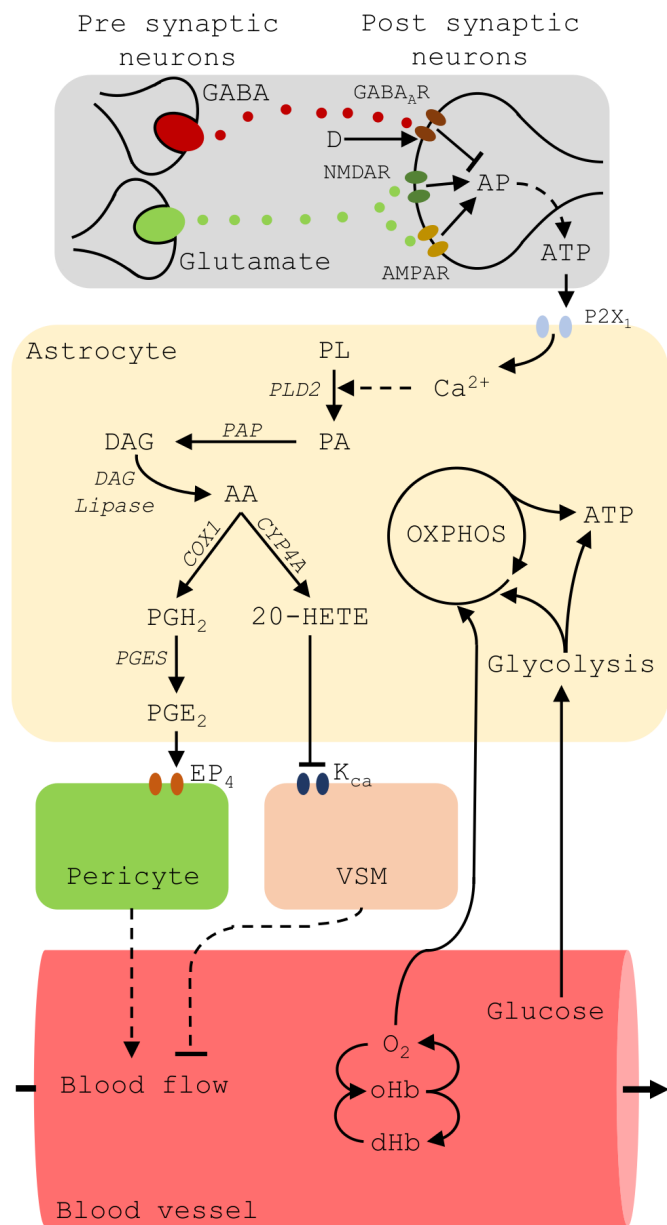

Figure 2.2: Representation of the neurovascular coupling, adapted from Paper IV. Glutamate activates $\mathrm{N}$-methyl-D-aspartate receptors (NMDAR) and $\alpha$-amino-3-hydroxy-5-

methyl-4-isoxazolepropionic receptors (AMPAR), resulting in an influx of cations. $\gamma$-aminobutyric acid (GABA) activates $\mathrm{GABA}_{A}$ receptors $\left(\mathrm{GABA}_{A} \mathrm{R}\right)$ and results in an influx of anions, thus counteracting the effect of glutamate. The balance between GABA and glutamate determine the probability of action potential firing. Generation of an action potential triggers ATP release, which activates $\mathrm{P}_{2} \mathrm{X}_{1}$ receptors in adjacent astrocytes resulting in $\mathrm{Ca}^{2+}$ influx. This generates arachidonic acid (AA) through the following three steps: 1) phospholipids (PL) form phosphatidic acid (PA) via $\mathrm{Ca}^{2+}$ activated phospholipase D2 (PLD2); 2) $P A$ is transformed into 1,2-diacylglycerol (DAG) by PA phosphatase (PAP); 3) $D A G$ is transformed into $A A$ by DAG lipase.

AA is metabolized into prostaglandin $\mathrm{H}_{2}\left(\mathrm{PGH}_{2}\right)$ by cyclooxygenase 1 (COX1) and consecutively into prostaglandin $\mathrm{E}_{2}\left(\mathrm{PGE}_{2}\right)$ by PGE synthase (PGES), or, into 20- hydroxyeicosatetraenoic acid (20-HETE) by cytochrome p450 4A (CYP4A). $\mathrm{PGE}_{2}$ relaxes pericytes via activation of prostaglandin $\mathrm{EP}_{4}$ receptor, promoting blood vessel dilation. 20-HETE contracts vascular smooth muscle (VSM) cells via inhibition of calcium activated potassium channels $\left(\mathrm{K}_{C a}\right)$, promoting blood vessel constriction. ATP is generated by glucose and oxygen metabolism via glycolysis and oxidative phosphorylation (OXPHOS). 
steps, leads to an increase in arachidonic acid (AA) [33][35]. AA is transformed into several vasoactive substances such as prostaglandin E2 (PGE2), epoxyeicosatrienoic acid (EET), and 20-hydroxyeicosatetraenoic acid (20-HETE) that control the blood vessel actions through intracellular parallell processes, thereby changing the blood flow and causing the post-stimulus peak and the post-peak undershoot of the BOLD response.

\section{Glutamate and GABA}

Activation and inhibition of neurons in the brain is controlled by neurotransmitter substances. The two most common neurotransmitters, and the only ones considered in this thesis, are glutamate and $\gamma$-aminobutyric acid (GABA). Their signal transmission can be seen in Fig. 2.2. Glutamate binds to N-methyl-D-aspartate (NMDA) and $\alpha$-amino-3-hydroxy-5-methyl-4-isoxazolepropionic (AMPA) receptors in the post-synaptic cell. These receptors depolarize the cell membrane, activating voltage-gated VDCC channels, which in turn open to allow $\mathrm{Ca}^{2+}$ to flow into the cell and trigger an action potential. GABA, on the other hand, binds to $\mathrm{GABA}_{A}$ receptors which increase the inflow of $\mathrm{Cl}^{-}$into the cell, which instead hyperpolarizes the cell membrane and impede the triggering of action potentials, thereby inhibiting activation in the post-synaptic cell.

Most neurons release mainly one of these neurotransmitters. Neurons that release mostly glutamate are called glutaminergic neurons and those that release mostly GABA are called GABAergic neurons. If we consider only the interaction of a pair of isolated pre-and postsynaptic neurons, glutamate is activating and GABA is inhibiting neuronal excitation. However, there must first be an action potential in the presynaptic neuron in order for it to release GABA. This means that the GABAergic neurons can be excited, even though the area of the brain that they belong to is generally inhibited. Furthermore, an activation of GABAergic neurons might, according to the neurotransmitter hypothesis, still trigger the intracellular signaling pathways leading to a BOLD response. Due to this, it is worth keeping in mind that the terms "activation" and "inhibition" do not correspond to all neurons in that area being excited or inhibited at the same time.

\section{The Negative BOLD Response}

The classical BOLD response have a significant rise and positive peak of the fMRI signal a few seconds after stimulation, and for a long time that has been the standard indication of brain activity in fMRI. However, this is not the only consistent signal change noted in many experiments. In several studies, areas with a negative correlation to the classical BOLD response are identified. In several of these studies, the negative correlation came from the fMRI signal decreasing below baseline 


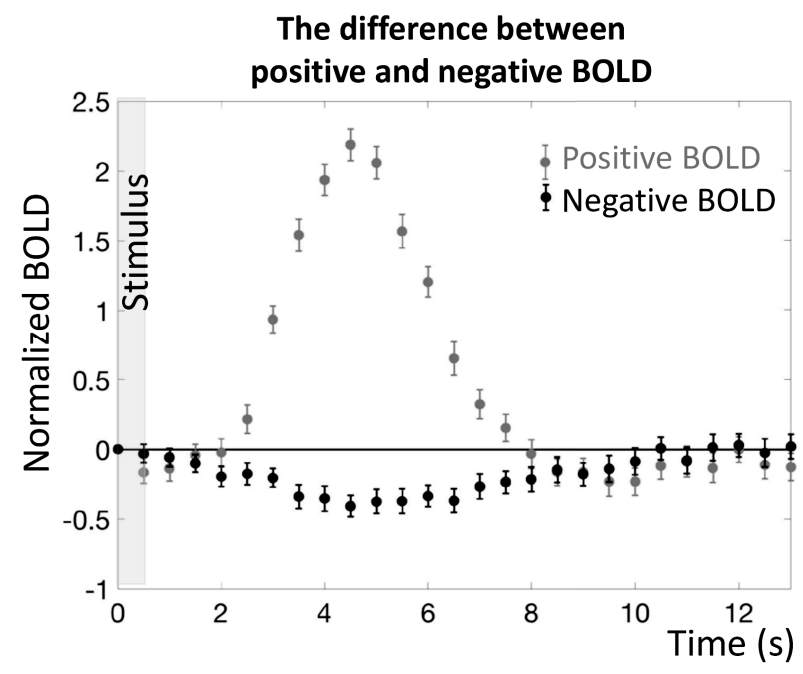

Figure 2.3: Data collected from the visual cortex showing a positive BOLD response (grey) and the posterior cingulate cortex showing a negative BOLD response (black).

during the task [36][37][38]. If the fMRI signal goes below baseline in response to a stimulus, it is called a negative BOLD response. In many fMRI studies, the negative BOLD response has not been taken into account in the final conclusions, or simply not been reported at all, as it has been unclear what the mechanisms behind the negative BOLD response are. One popular hypothesis has been the vascular steal hypothesis, stating that since the positive BOLD response reroutes an increased amount of blood into one area, that blood must be taken from somewhere, and therefore there will be areas with a lower signal caused by the active areas "stealing" the blood from their surroundings [39][40][41]. However, there are also studies showing a consistent negative BOLD response in areas which are ipsilateral to activated areas, and therefore unlikely to be explained by hemodynamic steal [42][43]. Another hypothesis is that an uncoupling of the oxygen metabolism and the regulation of $\mathrm{CBF}$ and $\mathrm{CBV}$ can cause a negative BOLD if the oxygen metabolism increases in response to increased neuronal activity while the $\mathrm{CBF}$ and $\mathrm{CBV}$ remain the same [44][45]. Neither of these hypotheses define the negative BOLD response as relevant in terms of extracting information about the level of neuronal activity. However, there is a hypothesis which strongly connects the negative BOLD response to neuronal activity levels, and that is the neural inhibition hypothesis. The neural inhibition hypothesis is based on studies 
that show decreases in both oxygen metabolism and blood flow correlated to negative BOLD responses [46][47][48][49], as well as decreased local field potentials and spiking activity in association with negative BOLD responses [47][50][51]. Together, these results points to that some negative BOLD responses are caused by inhibition of the neuronal activity [46][52][53]. Inhibition seems to be as important for the proper functioning of the brain's networks as activation is, and therefore the interest in the negative BOLD response has been rising lately.

\subsection{The Importance of Inhibition}

Inhibition in fMRI is referred to as a dampening of the brain activity in an area, sometimes down to baseline activity for the area and sometimes even below baseline activity. Inhibition seems to be an important mechanism for switching between different networks that perform different functions in the brain. The same brain area can be active in the several different networks and perform different functions depending on which network it is currently engaged in. Hence, it is necessary for the brain to regulate which areas that are active simultaneously, in order to avoid interference between different networks. The regulation of brain activity can be done by inhibiting areas that should not part-take in the currently active networks. An example of this was shown by Sridharan et al. [2], in a study on healthy volunteers where the interaction between three networks was investigated. One network was the Default Mode Network (DMN), a network that is most active when we let our thoughts wander and focus internally or try to understand the emotional state of another person. The other network is the Central-Executive Network (CEN), which is often active when when we are performing a task that demands focus and is often responsible for the control of attention and working memory. The third network is the Salience Network (SN), which in Sridharan et al.s study was found to control the switching between the DMN and the CEN depending on if the test subjects were performing a task or resting. Most notably, while focusing on a task, the DMN was inhibited. This is supported by findings from a study by Jilka et al. [54], which found that patients with lesions in areas important to the $\mathrm{SN}$ had slower information processing speed, reduced cognitive flexibility as well as it being harder for them to refrain from action compared to healthy controls. These difficulties in performing a task which required focus were accompanied by a failure to inhibit the areas normally active in the DMN. 


\subsection{Pharmacological Modulation of GABA}

In the clinic, MRI is used to examine a wide spectrum of diseases, disorders and traumas. One of the most important factors for getting high quality data during these examinations is that the patient lies still in the scanner. Sometimes this is difficult, the patient might e.g. be anxious about the result of the scan, or have claustrophobia and feel that the space in the scanner is uncomfortably small, or have some disorder that makes them want to move a lot. Small amounts of movement artifacts can be filtered away during the preprocessing of the data (see section2.8), but if the movement artifacts are too large, the whole data set might need to be discarded. Therefore, it is important to facilitate for the patients to lie still. Diazepam is a benzodiazepine that binds to the GABA receptors and increase the frequency of the opening of the $\mathrm{Cl}^{-}$channels, thereby increasing the hyperpolarization of the cell membrane and inhibiting neuronal activity in the post-synaptic neuron [55][56][57].It acts as a mild sedative that can alleviate angst and spasms, and work as a muscle relaxant.If a patient is anxious about being in the MRI scanner, diazepam can be administered in a low dose to help them calm down. However, as diazepam clearly has an effect on the mental state of the patient, it has been suggested that it might be inadvisable to administer to patients and test subject undergoing an fMRI examination as there is a risk that that it can alter brain activity. This is investigated in Papers III and IV, where we was found that for healthy volunteers who had $>0.01 \mathrm{mg} / \mathrm{L}$ of diazepam in the blood plasma, the BOLD response had indeed changed in the cingulate cortex. Instead of a positive BOLD response, which is commonly interpreted as an activation, the BOLD response had decreased under baseline and became negative. Since a negative BOLD response is often interpreted as inhibition, we concluded that the sensitizing effect of diazepam on the GABA receptors had caused the BOLD response to change its shape.

\subsection{The Structure of fMRI-Images}

Now it is time to turn our attention to the more technical aspects of fMRI data collection and analysis. fMRI images are a rather complex type of data, and understanding the different steps of the analysis methods used on them requires some attention being paid to the way the images are structured. The different levels of scale of an fMRI image is shown in Fig. 2.4A. The signal in an fMRI image is generated by the nuclear magnetic spin of the protons in a magnetic field, and as the signal is measured, the scanner also registers the localization of the signal in small volume elements called voxels. The spatial resolution of an fMRI image, or the size of each voxel, is usually $1.5-3 \mathrm{~mm}$ on each side. The resolution of an MRI 
A

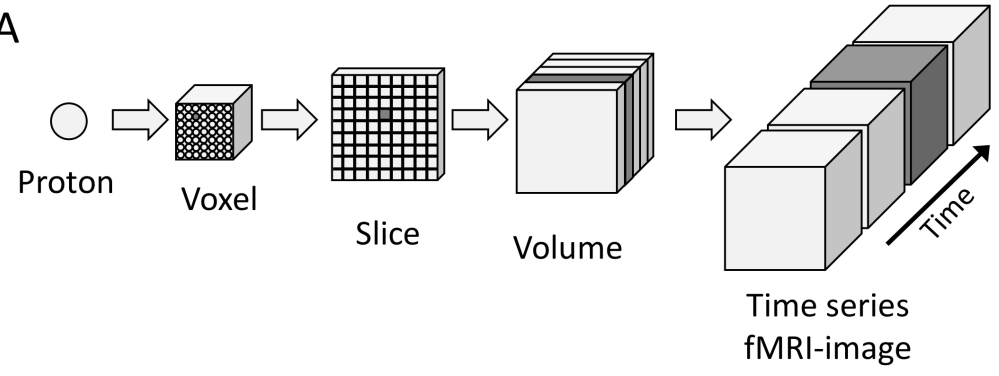

B
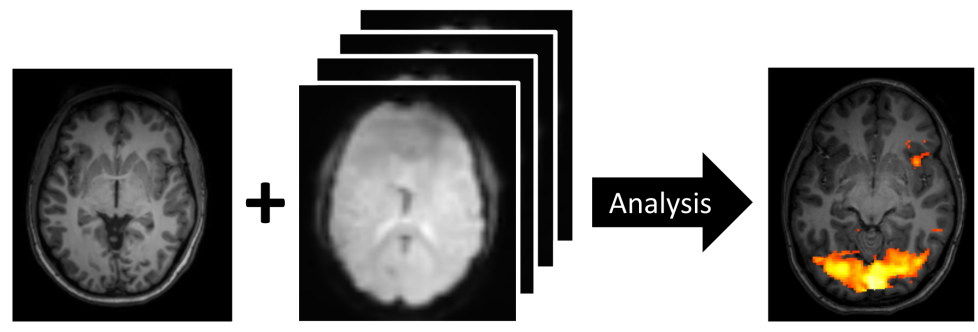

Anatomy image

fMRI images

Activation map

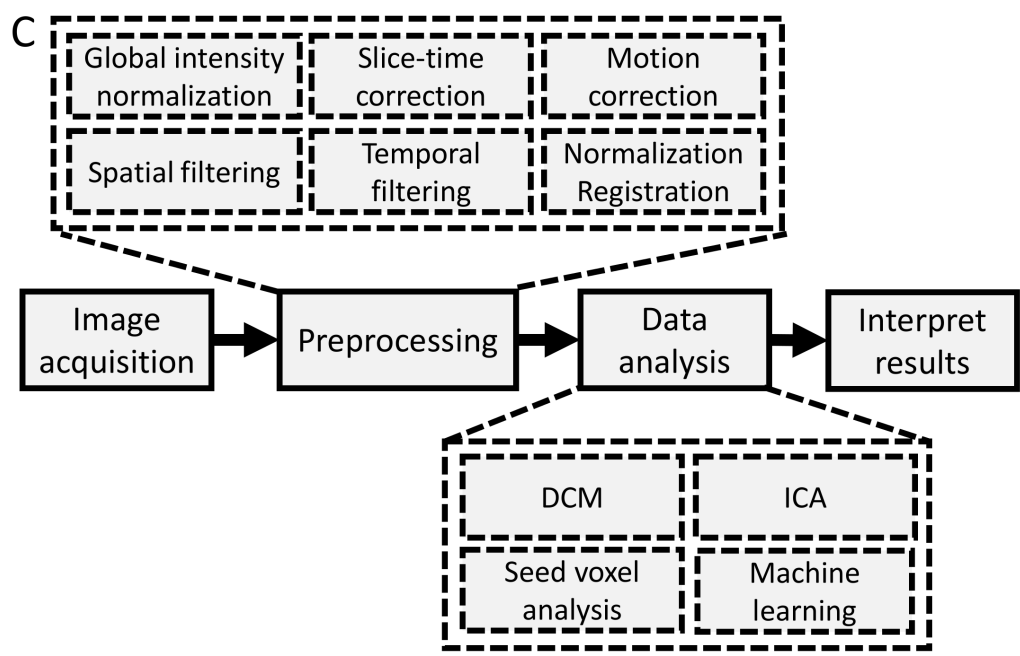

Figure 2.4: A. The building blocks of an fMRI image. B. Anatomy image and EPI image are measured in the scanner and combined in the analysis to create a map of the activity. C. fMRI workflow (full squares) and examples of methods which are commonly used (dashed squares). 
scanner is not enough to measure individual neurons. In a single voxel, there can be several types of tissue present, and the fMRI signal therefore originates from a mix of blood, brain tissue with several different types of cells in it, and cerebrospinal fluid. This diversity in signal source is referred to as the partial volume effect. The scanner measures one layer of voxels at a time in two-dimensional slices, which are then merged into three-dimensional images, or volumes. When we are only interested in the anatomy, one such volume is enough to give us the desired information, if the resolution is high enough. But in fMRI we are interested in changes over time, and an fMRI image therefore consists of several volumes measured consecutively for several time points. In theory, any such measurement of changes over time can be called fMRI, but in practice the term usually refers to the measuring of brain activity using the BOLD contrast.

\section{Anatomy Images and fMRI Images}

The MRI scanner has the ability to register many different kinds of images depending on the settings chosen by the researcher. Different examinations calls for different types of images, and the two types of images used in most fMRI studies are anatomy images and fMRI images. Examples of these two types of images can be seen in figure $2.4 \mathrm{~B}$. The anatomy image consists of only one volume and therefore has no temporal resolution. Instead, anatomical images have a high spatial resolution and are used as a reference to identify the area of the brain with increased activity. In the studies that make up this thesis, the anatomy images are T1-weighted, since these have a high resolution. Also, the grey matter is grey, white matter is white and cerebrospinal fluid is black, making it easy to recognize anatomical structures in the brain. fMRI images, on the other hand, have a lower spatial resolution and a temporal resolution typically of two or fewer images per second. It is the fMRI images that are used to analyze the change in the fMRI signal indicating brain activity. The fMRI images we used were T2* weighted, since such images are particularly sensitive to the type of signal changes that are used in the analysis. The two types of images are then combined in the analysis and the end result is typically represented as statistical heat maps, which are then superimposed on the anatomical image like the one in Figure 2.4B. The different colors represent statistical significance, interpreted as different levels of activity.

\section{7 fMRI Noise}

fMRI data is often noisy. fMRI noise stems from multiple sources and is neither white, nor normally distributed. However, in the simulated data in Paper II, we use normally distributed noise, since that is the underlying assumption of the analysis. 
Noise in fMRI data is often divided into thermal noise, system noise, physiological noise and random neural activity.

- Thermal noise is caused by a rise in temperature as the scanner is used. When the temperature rises, the molecules gain more energy and starts to move more and this is measured by the coils and introduce noise in the signal.

- System noise is noise caused by the scanner hardware. It can be e.g. drift or inhomogeneities in the magnetic field, signal loss caused by differences in composition in different parts of the brain and skull.

- Physiological noise is caused by processes in the body, e.g. such as breathing, heart beats and motion. According to [58], physiological noise is the main source of noise in fMRI and approximately $60 \%$ of the physiological noise comes from changes in $\mathrm{CBF}, \mathrm{CBV}$ and oxygen consumption, i.e the same mechanisms that control the BOLD response.

- Random neural activity is all the neural activity in the brain which is not connected to the function currently being measured, but which might interfere with it.

Differences in strategy between different test subjects or between different tasks in the same test subject might also confound the signal, although it is not strictly noise.

\subsection{Preprocessing of fMRI Images}

The prevalence of noise in fMRI data has driven the development of several methods to preprocess the images before analysis. There are many different strategies one can employ to reduce noise (see Figure 2.4C), but which combination of strategies is advisable is discussed. Too much modification of the raw data introduce the risk of changing the signal enough that the true BOLD response is lost, or artificial activity is introduced.

Some of the different pre-processing steps that can be applied are:

- Motion correction: Since human test subjects are living beings, they are unable to lie completely still during an entire session of scanning, as they breath and shift their position for comfort. However, the scanner will always measure the same grid of voxels relative to the scanner position and assume that the signal in the same voxel comes from the same location in the brain throughout the entire scan session. As the subject moves, their 
brain will shift position compared to the scanner, and therefore the fMRI signal for the same piece of brain tissue will in fact move between voxels. This might cause motion artifacts in the fMRI signal of the same amplitude as the BOLD response itself. The best way to prevent motion artifacts in the data is to make the test subject as comfortable as possible during the scan. Motion correction is a noise reduction method that uses one of the volumes as reference and then realigns all the other volumes to that same position. Motion correction algorithms can counteract small shifts in position, but if the movement is too big the data becomes useless.

- Slice-time correction or realignment: Since fMRI images are collected slice by slice, the different parts of an fMRI volume will be collected at different time points compared to the stimulus, which may affect the shape of the measured BOLD response. Slice-time correction puts the slices back in the correct order and attempts to show what the data should have looked like if it was all collected at the exact same time point.

- Spatial filtering or smoothing: To minimize the occurrence of false positives, different filters are used in order to average the value of a voxel with that of its neighbors. This increases the signal-to-noise-ratio and improve the quality of the data by reducing the variance, but it might also inadvertently erase low activation.

- Temporal filtering: High pass and low pass filters as well as Kalman filters can be used to filter out physiological noise and scanner drift, but should be used with caution as they might mask or erase the relevant components of the signal as well.

- Normalization or registration: All individuals are anatomically different, which complicates comparison between individuals and between groups. During registration, the volumes of each individual are mapped to a template brain image. There are several different types of transformations which can be used for the mapping, but each of them includes some kind of smoothing and risk introducing artifacts or obscuring the signal in the data.

\section{9 fMRI Experiment Design}

When designing experiments in fMRI studies, there are several important things to keep in mind in order to get the best image quality and activation relevant to the research question. fMRI experiments can be divided into categories, and here I will focus on the category that includes some kind of stimulus, as that is the type of data that the models presented here are used on. 


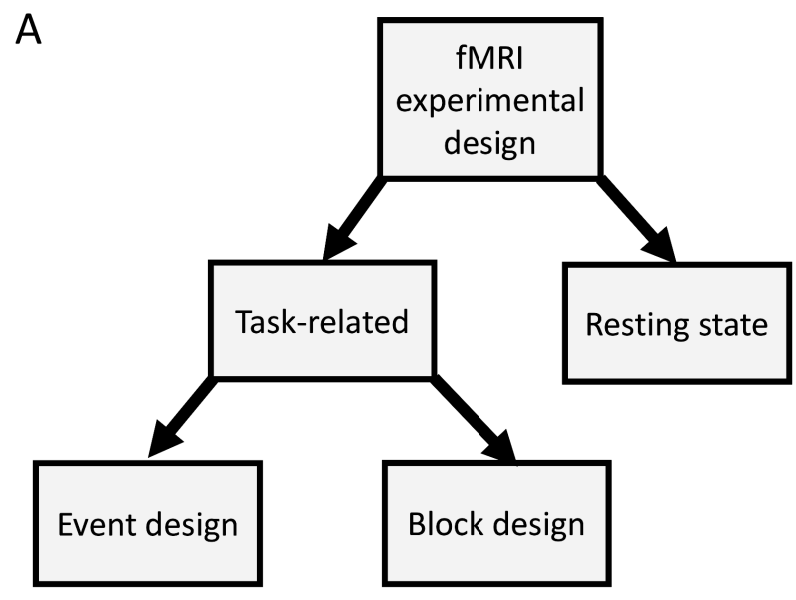

B

Stimulus
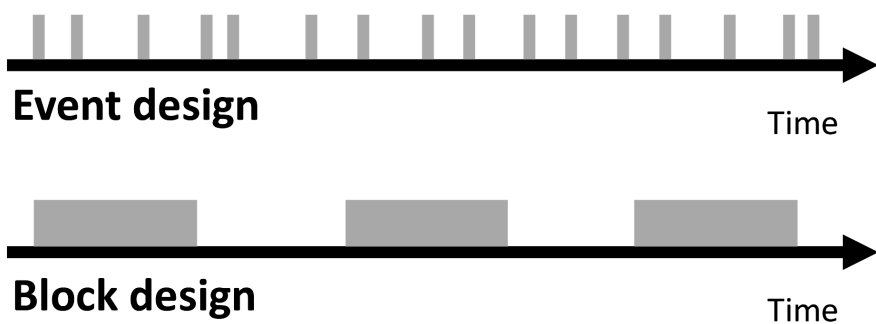

Figure 2.5: A. Categories of fMRI experimental design. B. The difference between event design and block design. An event design have short stimuli and long ISI compared to the stimulus time. In the analysis, each stimulus is treated as a separate event. A block design consists of periods of stimulation and long periods of rest which are considered in blocks in the analysis. 


\section{Tasks and Resting State}

One way of categorizing experiments is according to the type of brain function being investigated. The main categories are task-related fMRI and resting state fMRI, as shown in Figure 2.5A. In task-related fMRI, the test subjects are given a task to perform, which is constructed to engage specific functions in the brain, such at looking at pictures in order to activate the visual cortex or performing working memory tasks in order to activate memory-related brain networks. In resting state experiments, the test subjects are instead instructed to let their mind wander. This type of experiments aim to investigate different types of networks which are continuously employed by the brain, but often suppressed when we focus our attention on a certain task.

\section{Event Related Design versus Block Design}

Within task related experiment designs there are two main categories of designs, event related designs and block designs, illustrated in 2.5B. In an event related design, the stimulus is short and the interstimulus interval (ISI, the time before the next stimulus) is long compared to the stimulus, usually 2-20 seconds. A block design instead is divided into periods called blocks, which usually last 15 $30 \mathrm{~s}$. During a block, the test subject can be instructed to perform a task or to rest. Task blocks are either one long, ongoing stimulus or several stimuli with short ISI and the resting blocks have no stimulus at all. Blocks of one task can be alternated with blocks of another task or with blocks of rest.

\section{Experiment optimization}

It is not trivial to find the combination of experimental conditions that will result in a well designed fMRI experiment. There are three main categories of choices to make in each experimental setting. The first choice concerns the scanner settings. What type of images are most appropriate for what we want to measure? Do measure the whole brain or is it better to only image a few slices in order to speed up the image acquisition? In that case, what part of the brain should we measure? The next choice is what type of experimental design to choose. Taskrelated or resting state? If we want to image a specific function, a task is often chosen, and this task must naturally be one that activates the function of interest. Should the task then be given in an event or a block design? How long time should pass between every stimulus? Many of these questions are also related to the third choice, which is how to analyze the data. This means that the data must fulfill certain statistical assumptions, which might e.g. require a sufficient number of repetitions of stimuli or a minimal number of test subjects. We must also consider 
that it is humans that we are scanning and that the task must be designed taking into account human emotions such as boredom, sleepiness, habituation, anticipation and inability to keep focus for long periods of time. Tools that might be used in order to design the optimal experiment is repetition, randomization and jittering of stimulation type or ISI, as well as alternating between different tasks or between task and rest.

\subsection{Analysis of fMRI Data}

The "f" in fMRI stands for "functional" and expresses the desire to not only image the anatomy of the brain, but also its function. The key to this information lies in measuring changes over time. However, the brain performs several functions at the same time and it is not always easy to know which changes in the fMRI signal that are due to the task or function of interest, due to other functions that the brain performs, or simply noise. Since the brain fulfills many functions, many ways of analyzing fMRI data have been developed, and each of them serves a specific purpose for investigating specific types of brain function. The work presented in this thesis only concerns data collected from task-oriented fMRI experiments, so that is the type of analysis I will focus on describing.

Early methods in fMRI focused on functional specificity, that is, finding the specific centra for different functions in the brain. The first fMRI experiment and analysis were simple: periods of rest were alternated with periods of performing a task, the signal during rest was subtracted from the signal during activation and a t-test was used to identify a significant change in the fMRI signal [3]. Though effective to show the usefulness of fMRI in finding brain activation, the method only works for block designed experiments and it violates some of the basic statistical assumptions for the t-test [59].

The realization grew that if we were to understand the connection between neuronal activity and the fMRI signal, the shape of the BOLD response must be more thoroughly mapped. In 1996, Bruckner et al. [60] performed the first event-related experiments, where they used long ISI in order to capture the whole BOLD response, and then averaged the BOLD responses for many stimulations of the same kind (the experimental design in Paper I of this thesis is very similar to Bruckners design). At this point, the behavior of the BOLD response was intensely studied [28][46][47], and the discovery that for ISI $>2 \mathrm{~s}$, the BOLD response has a linear time-invariant system behavior [61][62][63], led to the development of the General Linear Model (GLM) which uses basis functions to simulate the BOLD response. In the late 1990s, an intense effort to reduce and filter noise from the fMRI signal also took place, forming much of the preprocessing methods now considered to be standard. 
In 1999, the SPM group [64] presented mixed effect modelling methods which greatly improved group analysis of task related fMRI data. However, this method was critizised for not taking intersubject variability into account in a proper manner, and therefore Woorich and Beckman developed FSL [65][66]. Both of these analysis programs have developed since then and are now staple in fMRI analysis.

The methods developed so far had focused on mapping the activity in specific regions of the brain related to different tasks, but interest for investigating networks and interactions between brain areas grew. This gave rise to several new methods for investigating connectivity and interactions, commonly referred to as functional integration methods [67]. These methods are based on theories from fields such as component analysis, machine learning and graph theory approaches. These methods focus on investigating two types of connectivity between brain areas, either functional connectivity that describe the temporal correlation of the BOLD signal between different areas (e.g independent component analysis [68]), or effective connectivity that searches for causal influences between neural systems (e.g. dynamic causal modelling [69]). Commonly used examples of these methods are shown in the lower dashed squares of Figure 2.4C. fMRI analysis is today spread out on a scale from hypothesis driven analysis methods, which relies heavily on modelling, to data driven methods, which are model free. In hypothesis driven fMRI analysis most methods are based on different kinds of models. Most of these models are very good at finding the shape of the BOLD response but lack a firm basis in the underlying biological mechanisms of the neurovascular coupling, and none of those that attempt to describe the mechanisms are based on the neurotransmitter hypothesis. Therefore we suggest a new type of analysis, based on methods taken from the relatively young field of systems biology in order to study the neurotransmitter hypothesis.

\subsection{The fMRI Information Loss Problem}

The fMRI signal is a secondary measurement of brain activity, and several factors in the brain and in the scanner system contribute to changes in the shape and in the temporal resolution between the neuronal signal and the fMRI images (illustrated in Figure 2.6). These factors induce lag, smoothing, inference, and both temporal and spatial noise into the fMRI signal, and therefore, some of the information about the activity on a neuronal level is lost or obscured. This has been one of the main causes for critique against using fMRI data in order to draw too detailed conclusions about brain function [16]. Today this information loss problem is dealt with using phenomenological approaches that compare the signal from the fMRI camera with basis functions describing typical behavior observed in response to activity. This ignores and does not make use of our understanding of the under- 


\section{Sources of fMRI information loss}

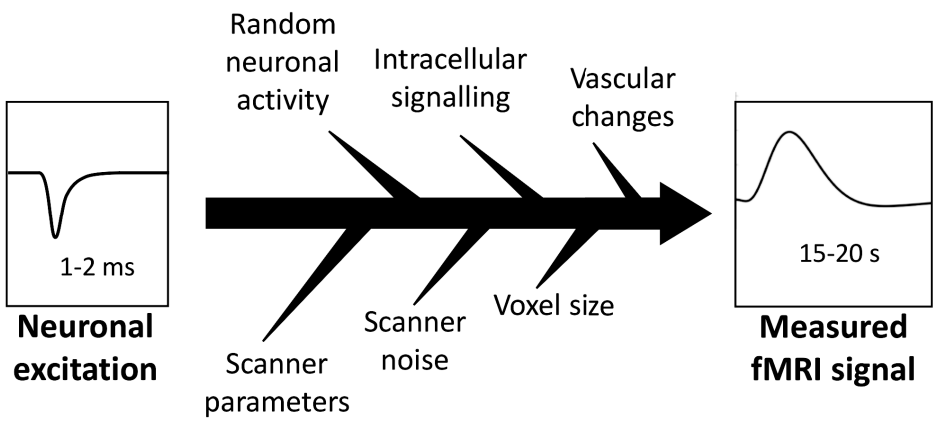

Figure 2.6: Sources of fMRI information loss.

lying biology. Mathematical modelling is a potential way to introduce biological knowledge back into this data analysis. There are mathematical models developed, describing the interplay between blood volume and blood flow. However, mathematical mechanistic models based on some of the most commonly believed mechanisms for the entire neurovascular coupling, such as the neurotransmitter hypothesis, has to date not been neither developed, nor applied to fMRI data, and their potential to reverse the information loss problem is therefore still unexplored.

Here follows a short summary of the main sources of information loss in fMRI.

- Blood flow and blood volume both affect the fMRI signal. These are fundamental mechanisms of BOLD measurements, but they also have several confounding effects. Firstly, vascular changes caused by neuronal excitation are slow compared to the neuronal signal, and therefore introduce delay and smoothing of the signal. Secondly, as blood moves through the blood vessels, the fMRI signal might originate from downstream effects, rather than at the true site of activity. Thirdly, the changes in blood flow brings in oxygenated blood, which might mask the effects of oxygen metabolism on the fMRI signal.

- Partial volume effects because the voxel size is bigger than the neurons.

- Unrelated activity in the brain which might interfere with the function of interest.

- Intracellular signaling may change the shape of the signal and introduce delay. 
- Scanner noise and scanner baseline drift.

- The scanner parameters chosen by the technician, such as e.g. the time between the measured time points [70].

The loss of information is a problem if we want to use fMRI more extensively than we do today in order to measure and study brain networks. The question is, how much information still remains in the images and time curves obtained from the scanner, and how do we analyze that information in order to use it to its fullest potential? In this thesis we demonstrate a modelling framework which has the potential overcome some of the information loss we face today. 
"The beauty of a living thing is not the atoms that go into it, but the way those atoms are put together."

Carl Sagan, Cosmos 


\section{Chapter 3}

\section{Systems Biology}

\subsection{System Properties}

System properties, or emergent properties, are properties that emerge through the interactions between the components of a system, and can not be detected in the isolated components. A classic example of a system property is surface tension, which can not be measured in a single water molecule, but only when lots of water molecules bind to each other. The way that flocks of birds or school of fish move in concert by each individual following a very simple set of rules are also examples of emergent properties of the system, as is crosstalk in intracellular signal transduction. Indeed, many properties in nature are system properties. Because these properties can only be observed when the system is complete, they are often hard to study. The more components and interactions a system has, the more complex it becomes, and more advanced methods of analysis are required to study it. However, the gain from this type of studies is great, since system properties contain a lot of information about the system. Therefore, to study the system as a whole gives a more holistic view, e.g. of how to treat a patient.

\subsection{What Defines Systems Biology?}

Systems biology is a term with many different definitions, but all of them are based on a holistic worldview. In this thesis, I refer to the field of systems biology as an interdisciplinary field where biology, mathematics and, computational science are employed in an effort to find more holistic ways of analyzing biological systems [4][71]. It relies heavily on computational models, because the strength of computers is that they can store huge amounts of data and quickly perform calculations, which makes it possible to study whole systems at once. Systems biology is often presented as the opposite of reductionism, which is focused on identify- 
ing the properties of isolated components in a system, rather than investigating how these components fit together. The basis of a systems biology workflow is to collect many types of data from different parts of the system and then use mathematical models to analyze the interactions of the measured components. This strategy gives rise to new opportunities to investigate and predict system properties. One essential tool in systems biology is mechanistic modelling, which will be further described in section 3.3.

\subsection{Modelling Biology With Mechanistic Models}

There are many ways of building models describing biological systems, and each type of model serves a different purpose. As many biological systems are big and complex with many interactions and high levels of measurement noise, models have for a long time been a necessary tool to understand them. A model is always a simplification of the system and can never fully describe reality, but by choosing intelligently how we construct the model, the system can be investigated at different levels of detail. Here it is important to realize that all models lie in a scale from phenomenological to mechanistic [4]. Phenomenological models (also called black box models) are built from equations that describe the behavior of the system but have no biological ground. Like the basis equations of the GLM, they only describe the shape of a known curve. Mechanistic models (or white box models) on the other hand focus on describing the underlying system that the data is collected from. In a pure white-box model, each equation represents a biological mechanism from the system of interest, i.e. the model is fully mechanistic, and all parameters have been measured and are assumed to be known. Most models lie on a scale between these two extremes, and which degree of realism is the best to choose depends on what you want to use your model for. There are many factors determining what is appropriate, such as what level of realism that is desired, how difficult they are to compute and how we want to be able to interpret them. Always look to the use of the model in its context. Too phenomenological and it does not give the desired information, too complex and it might not be possible to interpret it. How much computational power is available? Who will use the model? What data can be used to validate that the results are reasonable? Mechanistic models enables us to look at the whole system and understand consequences of interactions, rather than investigating single components. Using such models, it is also possible to zoom in time and space if desired, and to predict results of experiments which are not possible to perform [4]. Mechanistic modelling also forces us to be more rigid in the description of the hypothesis in order to formulate the equations, and this in turn highlights gaps in knowledge [4]. Phenomenological models are usually cheap to produce in terms of time and computation but instead 
requires lots of data, while mechanistic model production is comparatively more expensive but offers the possibility to tailor experiments using iterative study design (explained below). Mechanistic models also yield more information about the system. However, working with mechanistic models typically requires a lot of computing power, and until the recent development of increasingly powerful computer systems, the usefulness of models have been limited by what we can calculate by hand. This can be exemplified by figure 12 in Hodgkin and Huxley's article [9], which presents one of the earliest and most famous mechanistic models in the field of neurobiology. Out of the three curves in the figure "Only one [...] is complete; in the other two the calculation was not carried beyond the middle of the falling phase because of the labour involved." Today, it would be unacceptable to publish only half a curve, since computers are such a widely spread tool. As more and more powerful computers and methods have become available, it is possible to replace or enhance experimental procedures with mathematical models. This is helpful in reducing the number of test animals used in research, and is less environmentally harmful than many experimental setups [4]. They are also cheaper and easier to share between groups or use in the clinic, and can be run many times, producing stable and reliable results every time.

Mathematical modelling is a rapidly evolving field, as the constant development of computers have opened up completely new possibilities for demanding computations and data storage, and we are seeing an abundance of new methods in statistics and numerical methods taking shape, and can expect to see much more of it in the future as the discipline of mathematical modelling of biological systems is just beginning to stretch its new and powerful wings.

\section{Iterative Study Design}

One of the strengths of systems biology is its usefulness in iterative study design, where it offers a method for strategic planning of systems analysis and experimental design [71]. Figure 3.1 shows how such an iterative study design can be set up. The work process starts with a set of data and a biological hypothesis described in the literature, or by a fellow researcher in the relevant field. The hypothesis is then translated into equations and implemented in a modelling program. In the papers in this thesis, the SB toolbox for Matlab [72] has been used, as it offers multiple toolboxes for such purposes. After this initial step, we enter a cycle of model testing, modification, predictions and experiments. All the steps of this workflow are described in depth in Paper I and [73][74], but here I will present a quick overview of the general idea behind the workflow of model development.

The first step in the cycle is to fit the model to the data. If this is unsuccessful, the model is rejected. A rejected model is an indication that the hypothesis might be wrong. At this stage it is important to go over the equations to see if they are 


\section{Model development cycle}

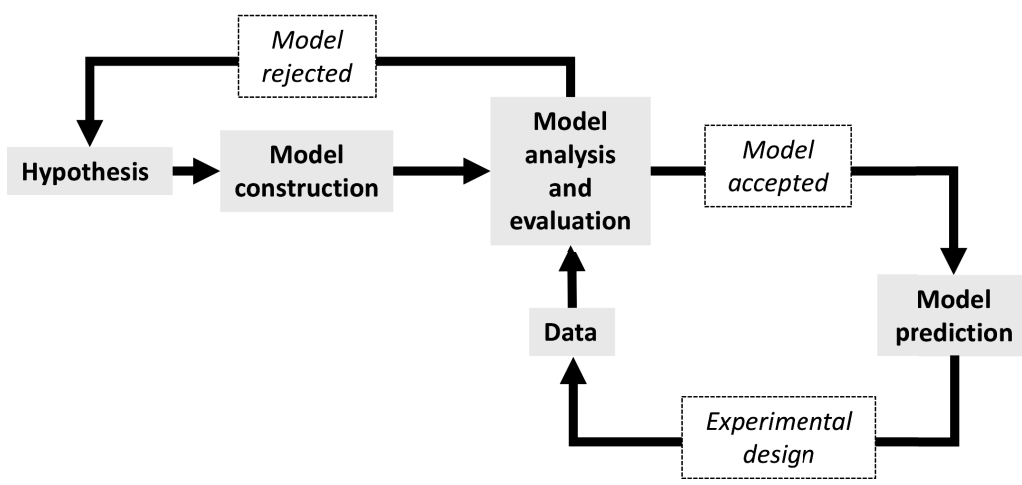

Figure 3.1: Workflow of iterative study design. Grey squares show the different steps of the iterative study framework and dashed squares show the possible outcomes of steps where the outcome effect the choices made during the next step.

an accurate representation of the biological system. If they are, and the model still can not be fitted to the data, the hypothesis needs to be revised or rejected. If the model can fit the data, the model is tentatively accepted. Several types of additional tests can be performed at this step, such as comparison to validation data and evaluation of behaviors of internal mechanisms which are not measured in the data. If the model all of theses tests, it is reliably accepted. Once the model is reliably accepted, the next step is to make predictions about systems behaviors. This can e.g. be how the system would react to new experimental conditions. These predictions may then be tested experimentally, new data collected and this new data is fed back into the model analysis step, starting the model testing cycle over again. Every time the model is analyzed it may be rejected, and indeed this happens quite often. In that case, a mechanism might be exchanged for an alternative one or a new mechanism may be added to test alternative interpretations of the hypothesis.

In summary, the two major benefits to the iterative study design is that for each turn of the modeling cycle 1, the biological hypothesis becomes stronger and more detailed, and 2, the experimental setup becomes more effective and focused. 


\subsection{Ordinary Differential Equations and Model For- mulation}

Ordinary Differential Equations (ODE) are a type of equations which describe rate of change. Since they describe rate of change, they are well suited for modelling dynamic processes in order to analyze time series data. They are commonly used for model construction in systems biology, and there are several reasons for this. One of the reasons why ODE models are so popular is because they can be viewed as a middle ground in many aspects of model construction.

ODE models in biology are often based on the Law of Mass Action, which means that instead of describing what each molecule does, they describe the mean reactions of the system. There are other types of models which are more detailed and complex in this aspect, e.g. stochastic models which contain mechanisms which introduce stochasticity and might describe the movement of each molecule in a system. Likewise, there are models which are better at describing spatial aspects than ODE models. It is possible to describe spatial differences in ODE models, but only if the species described in the model are separated into compartments. For more complicated relationships, such as e.g. concentrations gradients within the same compartment, partial differential equations (PDE) models might be a better choice. However, even though higher degrees of complexity opens possibilities to describe certain aspects of biological systems in more detail, it comes at a price. Increased complexity often makes the results of the model analysis harder to interpret and complex models normally have a higher computational cost and parameters which are potentially impossible to estimate. ODEs have a lower degree of complexity than both stochastic models and PDE models, but still higher than Black Box models. This makes ODE models cheap enough to compute, simple enough to understand, yet complex enough to bring nuance to the analysis. In view of these properties, it is easy to understand why ODEs are such a popular choice for modelling, not only in systems biology frameworks.

A more detailed description of how ODEs are used in the models presented here can be found in Paper I, [74] and [73]. In short, ODE models are centered around states, which usually represent the amount or concentrations of the entities in the model. A state can e.g. be the amount or concentrations of a molecule or metabolite, or the proportion of hemoglobin that is oxygenated could be one state while the deoxygenated proportion is another state. In the papers presented in this thesis, states are represented in a nondimensionalized manner, which means that the concentrations and amounts have arbitrary units [75][76]. This is often used when we do not know the real values for the concentrations or amounts for most components in the system. The advantage of nondimentionalization is that hypotheses and mechanisms are valid for all combinations of concentrations 
and units and not only for a specific combination of values. However, this also means that there is a risk of rejection caused by a lack of detail. When using nondimentionalization it is also important to be weary of overfitting (discussed further on), which can bring unrealistic parameter values.

In an ODE model, each states rate of change is described by an ODE. The ODE is comprised of rate reactions, where each rate reaction describes factors that increase or decrease the state. How much a state increases or decreases for a certain time step is decided by the values of parameters that are part of the rate reactions. These parameters are typically rate constants, whose values are often unknown and needs to be estimated. More on how to do that in the next section.

ODE models can not be calculated immediately for any chosen time point of the simulation. Instead, the model must be simulated by beginning at the first time point with a start value for each state, called the initial values. Then, using the rate of change specified in the ODE of each state, the value of each state after one time step is calculated. For the next time step the same calculation is repeated, but using the values that were just calculated as start point, and so on until all time steps have been calculated.

\section{Parameter Estimation}

The parameter values of mechanistic models are often unknown. There can be many reasons for this. They might for instance be impossible or unethical to measure with current measuring techniques in the system of interest. Similarly, it might be impossible to measure them under the same experimental conditions as the outputs and inputs of the model. Therefore, the values of the parameters are estimated through parameter optimization. There are many different kinds of optimization algorithms which employ different strategies for finding the best parameter values, but what they all have in common is that they compare how well different sets of parameter values can make the model fit the data. Model fit to data is measured by the cost, which in this thesis is defined by the cost function

$$
\begin{gathered}
V(\theta)=\sum_{\forall i, j}\left(\frac{\left(y_{i}\left(t_{j}\right)-\hat{y}_{i}\left(t_{j}, \theta\right)\right.}{\sigma_{i}\left(t_{j}\right)}\right)^{2}+\text { additional terms } \\
S E=\frac{\sigma_{i}\left(t_{j}\right)}{\sqrt{n_{i}\left(t_{j}\right)}}
\end{gathered}
$$

where $V$ is the cost, which is dependent on the initial conditions and the parameters $\theta, y$ is the measured value at the time $t$ and $\hat{y}$ is the corresponding value 
simulated by the model for those parameter values. $i$ is the number of datasets, $j$ is the number of data points and $\sigma$ is the standard deviation of the data, which is replaced by the standard error of the mean (SE, equation 3.2) when the data is the mean of many individuals.

The additional terms (also called weights or punishments) in equation 3.1 can be used in order to strengthen the influence of traits that might be important in the hypothesis, but which are not present in the data or add more weight to certain traits in the data. This could e.g. be the initial dip of the BOLD response, as is shown in Paper I. The additional terms are often formulated so that they are zero if the desired condition is fulfilled and otherwise they are larger the further away from the desired condition the model simulation is. Such additional terms are also used in the observability analysis in Paper II, but more on that in section 3.6.

The cost is based on the residuals $y_{i}\left(t_{j}\right)-\hat{y}_{i}$, which is the difference between the model output simulation and the measured data. The smaller the difference is, the better the model fit, the best fit being the global minimum of the cost landscape. The manifold surface made by the cost values from all different sets of parameter values is called the cost landscape, and the shape of the cost landscape can be used to tell us important information abut model properties. More on that in the section 3.6. The optimization algorithms used for estimating the parameter values employ different methods to search for the parameter values which results in the global minimum. There are many different optimization algorithms. The different kinds of optimization algorithms used in this thesis are particle swarm and simannealing. Particle swarmis a so called global optimization algorithm. It we envision the cost landscape as a landscape with valleys and hills, the global optimization algorithms can move anywhere in this landscape, wether the cost is high or low, which enables them to search for a good solution in a big part of the cost landscape.

Simannealing on the other hand, which is the Matlab function primarily used in this thesis, is a combination of a global optimization algorithm called simulated annealing, and a local optimization method called Nelder Mead Simplex method. Local optimizations usually have a start point in the cost landscape and move downwards in cost from there. This is a good way to refine the estimated parameter values, but if the start point is not well placed there is a risk that the optimization ends up in a local minimum instead of finding the global minimum. Therefore, global optimizations are often used in order to find good start points for the local optimization algorithms.

\section{Overfitting}

If a model has too many parameters compared to the amount of data available for fitting the model, a phenomenon called overfitting can occur. This is when 
the model is so flexible that it fits not only to the desired shape of the measured signal, but also fits to the noise or variation in the data in an exaggerated manner. If a model have a cost which is very close to zero despite noise being present in the data, it is appropriate to suspect that the model is overfitted. Overfitting increase the risk of faulty conclusions being drawn from the model analysis results. Overparametrization and overfitting is something one needs to be wary of in systems biology applications, as the models tend to be complex and quite large compared to the amount of available data. However, it is not as big of a problem as it could be, because the models are still not highly flexible, as they are based on the believed mechanisms, instead of flexible basis functions. In practice, there are methods for counteracting overfitting and using validation data is the best ways and most straightforward way to do that.

\section{Model Validation}

Validation data is data that have not been used in the estimation of the parameters. It can be data from a separate experiment or from individuals in the same experiment but which have been withheld from the estimation data. Validation data is a good way of discovering overfitting. If the model is overfitted, it will have a poor fit with a high cost when compared to the validation data. If there is not enough data during the parameter estimation to set some aside to act as validation data, predictions from the fitted model can be used to design the next experiment in the iterative study design cycle to provide validation data. It is also possible to use data provided by another lab, but then there is a risk of introducing additional uncertainty unless the experimental conditions are completely known.

\subsection{Model Minimization}

Model minimization is done for reasons of facilitating interpretation of the model analysis results, identifying essential mechanisms in the system, reducing the number of assumptions and decreasing the computational cost that comes with big and complex models. The aim is to find a model with as few components with as simple relationships with each other as possible, yet still able to explain all relevant data. Model minimization in the context of this thesis is made by taking away states and mechanisms one by one from the original model, reestimating the best parameter values and evaluate the model fit. When the model can no longer fit the data if any more states or mechanisms are removed, the minimal model has been found. This procedure is exemplified in Paper I. The model minimization must be done with regard to the scope of the model, as minimized models tend to become more and more phenomenological. Therefore one might not always de- 
sire to reach the minimal model, as this might in some cases take away the point of the mechanistic part of the modelling in systems biology.

\subsection{Model Uncertainty and Analysis of Identifiabil- ity and Observability}

Uncertainty in the results of the modelling analysis can come both from insufficient knowledge of the system, variation in the data and uncertain or unknown experimental conditions. All of these factors contribute to uncertainty in the estimated parameter values. Therefore, it is not as interesting to find parameter values that corresponds to the minimal cost, as it is to find a range of parameter values with an upper and a lower boundary for each parameter.
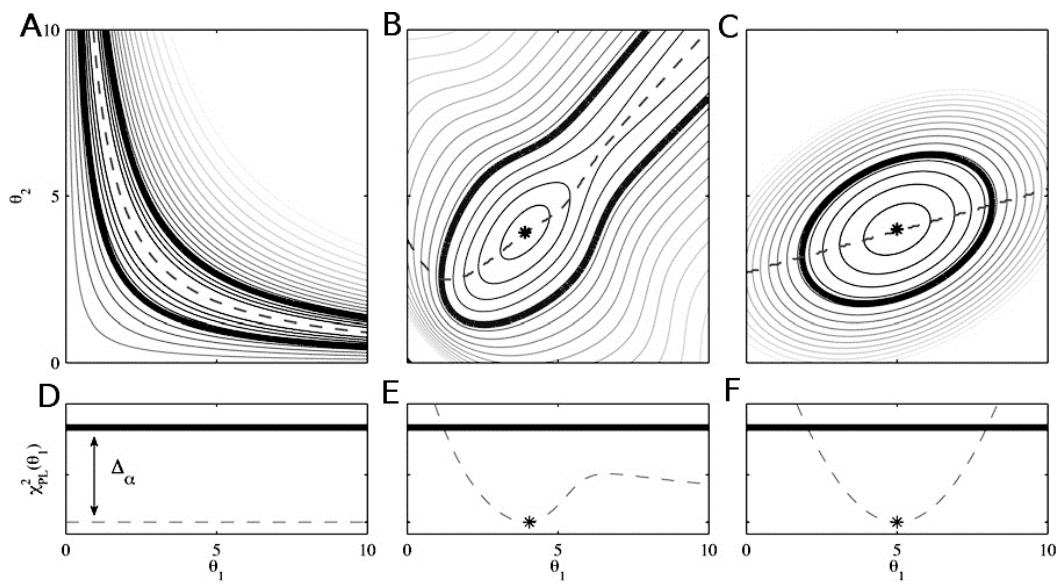

Figure 3.2: A-C. The cost landscapes of a two parameter system, D-F. Cost profiles corresponding to the same system. $A$ and $D$ shows a situation of unidentifiability, $B$ and $E$ shows semi-identifiability and $C$ and $F$ shows full identifiability. Dashed lines are the profile likelihood function and bold lines are the cost thresholds. Originally published as figure 4 in [77]. Included with permission from the authors.

Figure 3.2 A-C shows three different cost landscapes derived from two parameters, $\theta_{1}$ and $\theta_{2}$, in a hypothetical model. The solid curves indicate the contour of the cost landscape, and the thick, solid line represents a threshold of acceptable cost. The dashed line is the trace of the lowest cost as the value of the parameters increase or decrease. In plot D-F we can see the corresponding profiles for 
the cost for the parameter $\theta_{1}$ as $\theta_{2}$ is changed. This type of plot is called a profile likelihood plot, and is the result of a profile likelihood analysis [78][79]. In Figure 3.2 A and D, we can see in the profile likelihood plot that the cost is low and never go above the threshold. This is a case where the parameter $\theta_{1}$ is unidentifiable. Figure 3.2 $\mathrm{C}$ and $\mathrm{F}$ instead shows a case where the the parameter $\theta_{1}$ has an upper and a lower limit where it crosses the threshold. $\theta_{1}$ is then fully identifiable. The graphs B and E shows a case of semi-identifiability, where the parameter $\theta_{1}$ have a lower boundary but not an upper one. In this case, the parameter is practically unidentifiable, since the threshold can be lowered enough that the parameter also crosses the threshold for an upper value. Since the threshold is often set with regard to the uncertainty in the data, this can be achieved by using data with lower variation, if possible.

A model is said to be identifiable if it is possible to find a limited span of values for each parameter in order to fit the data. Sometimes it is not possible to identify single parameters, and this is often the case for large models or model with many interactions that may create mechanisms that counterbalance each other. Such models are unidentifiable.

Even though the true values of the model parameters might be unknown, there is still a lot of information to be gained both about them and about other model properties. For instance, knowing the uncertainty profiles for a model is important, since they define the situations in which it is reasonable to trust the predictions of the model. Unidentifiability brings a lot of uncertainty about the predictions of the model, but it does not render the model useless. It might be possible to estimate other model properties, such as the sum or ratio of two or more parameters or states. This quality is called observability. Both identifiability and observability provide basis for finding biomarkers in mechanistic models, as using these concepts is possible to investigate mechanisms or relationships which are otherwise not measurable. Observable properties in a systems biology model might, but are not required to, correspond to known biological mechanisms.

\section{Prediction Profile Likelihood}

Profile likelihood analysis can be used in order to map the cost profile of a parameter. In cases of unidentifiability, prediction profile likelihood (PPL) analysis can be used. These two types of analysis are very similar, and therefore we shall go through only the PPL, as that is the type of analysis used in Paper II. The profile likelihood is used in the same manner, but on single parameters instead of model properties.

PPL is a model analysis method aimed at finding observable model properties. A model property can be a biologically interpretable mechanism in the model or simply a relationship between parameters or states, that is, anything that can be 
quantifiably extracted from the model simulations. E.g., in Paper II, the influence on the BOLD response from the vasoconstricting and vasodilating branches of the model is investigated as a model property. The model property of interest is then stepped up and down by being forced to assume a certain value and at each step the parameter values are reestimated under that condition, and the cost evaluated using a modified version of the cost function

$$
V^{P P L}(\theta)=V(\theta)+w *\left|Z(\theta)-Z^{\prime}\right|
$$

where $V(\theta)$ is the original cost calculated with equation $3.1, Z(\theta)$ is the simulated value of the model property, $Z$ ' is the step value of the model property, and $\mathrm{w}$ is a weight with a large value designed to force the model property to take the value $Z$ ' by making the term

$$
\left|Z(\theta)-Z^{\prime}\right|=0
$$

\section{Fully observable mechanism}

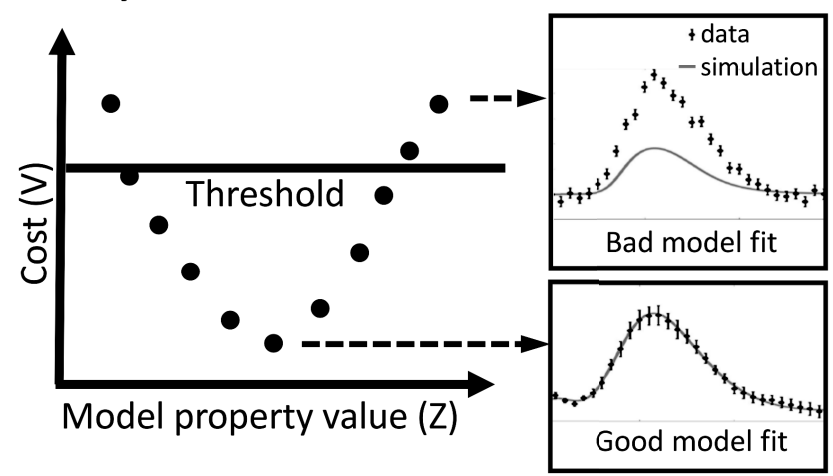

Figure 3.3: Schematic PPL graph of fully observable model property. Each dot is a re-estimation of the parameter values with the model property locked at the step value. The lower the cost, the better the fit of the model. If the cost of the model fit is above the threshold, the residuals are big and the model fit is bad, but at a low cost the residuals are small and the model fit is good.

A PPL graph, such as the schematic one shown in Figure 3.3, has the value of the model property on the $\mathrm{x}$-axis and the cost of the model fit on the y-axis. For each step of the PPL analysis, the model property is locked and the model is then fitted to the data and the cost is calculated. A threshold value for the model cost is 
decided for what is an acceptable model fit. If the model property is observable, there will be several steps when the cost is lower than the cut off, but eventually there will come a value of the model property when the model can no longer both keep the model property value at the locked value and fit the data at the same time. The model property value where the model cost cross the threshold is the limit of the span of values which the observable model property can have, corresponding to the identifiability analysis shown in Figure 3.2. A model property with an upper and a lower limit is fully observable, while a model property with only one limit is partially observable. If the cost of the model is always below the cut off, the model property does not have any limits to the values it can take and is therefore non-observable. If the cost of the model fit is always above the cut off, the model can not be fitted to the data and we have no information on the model property.

\section{Observability in Identification of Potential Biomarkers}

Both observability and identifiability are good methods for identifying potential biomarkers. E.g., Forsgren et al. [80] used mechanistic modelling and MRI images to estimate physiologically relevant and identifiable parameters that can be used in diagnosis of liver damage. The fact that there is a limited span of values which an observable model property can assume gives us a prediction of how the system ought to react. If the span differs significantly between groups, such as healthy and sick or between different diseases or disorders, then it can be used to separate these groups, even though the property itself can not be measured or is obvious to observe in the data. Using identifiability and observability for this purpose is a recently developed method, as this type of analysis is computationally heavy but has been made possible by new developments in computing. 
"An ultimate joint challenge for the biological and the computational sciences is the understanding of the mechanisms of the human brain, and its relationship with the human mind." 


\title{
Chapter 4
}

\begin{abstract}
Aims
The overall aim of the studies included in this thesis is to develop the basis for a new type of fMRI analysis based on principles from the field of systems biology. The founding principles of this analysis should be mechanistic modelling, predictions and testing on real data and clinical applications. It is my hope that in the future, this framework will be useful in the investigation of human brain function, particularly in network analysis, and in identification of biomarkers that can be used as tools for clinical diagnosis for brain diseases and disorders.
\end{abstract}

These are the specific aims for this thesis:

- Aim 1: To develop a mechanistic model of the neurovascular coupling, which can describe both activation and inhibition in fMRI data with focus on intracellular processes.

- Aim 2: To ensure that the model can explain the positive BOLD response as the result of activation and the negative BOLD response as the result of inhibition.

- Aim 3: To identify potential model-based biomarkers.

- Aim 4: To explain the impact of pharmaceutical modulation of GABA on the BOLD response. 
"It was on a dreary night of November that I beheld the accomplishment of my toils. With an anxiety that almost amounted to agony, I collected the instruments of life around me, that I might infuse a spark of being into the lifeless thing that lay at my feet."

Victor Frankenstein in Mary Shelleys "Frankenstein; or the modern Prometheus" 


\section{Chapter 5}

\section{Results}

\subsection{Aim 1: Model development}

In order for a model to be able to simulate a BOLD response, it must be able to simulate the main traits described in the litterature: for the positive BOLD response they are the initial dip, the post-stimulus peak and the post-peak undershoot, and for the negative BOLD response it is the signal decrease below baseline. In order for the model to be counted as mechanistic, it must be able to simulated these traits in a biologically plausibel manner. In Paper I, two different hypotheses of the mechanisms of the neurovascular coupling are tested (shown in Figure 5.1).

A Metabolic
feedback hypothesis

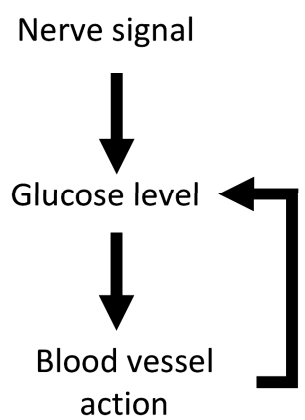

\section{B Neurotransmitter feed-forward hypothesis}

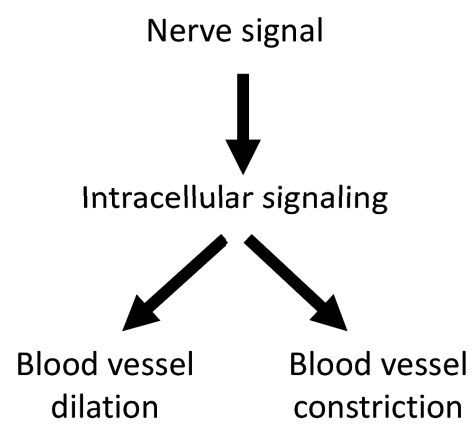

Figure 5.1: A. The metabolic feedback hypothesis. B. The neurotransmitter feedforward hypothesis. 
The first is the metabolic feedback hypothesis, stating that the increased blood flow forming the main peak of the positive BOLD response is initiated by a lack of either oxygen or glucose caused by an increased metabolism in response to increased workload on the neurons and glial cells. The second hypothesis is the neurotransmitter feedforward hypothesis where the BOLD response is initiated by intracellular signaling pathways which release vasodilatory and vasoconstricting substances controlling the blood flow. By modelling theses two hypotheses we could show several interesting properties of the system. Firstly, if the BOLD response was controlled by a lack of nutrients, it must be controlled by the level of glucose and not the level of oxygen, as the oxygen-controlled model was unable to create a post-stimulus peak. Furthermore, if the BOLD response was controlled by feedback from the metabolism of glucose and oxygen, the metabolism must be uncoupled between glucose and oxygen. This means that the metabolism becomes more, but not exclusively, anaerobic during stimulation in order to achieve both an initial dip and a post-stimulus peak. However, despite having both of these mechanisms, the model could not simulate the positive BOLD response in a biologically plausible way as the glucose level became depleted during stimulation (see Figure 5 in Paper I). The metabolic feedback hypothesis was therefore rejected. The neurotransmitter hypothesis could simulate both initial dip, poststimulus peak and the post-peak undershoot (see Figure 6 in Paper I). However, the initial dip was in the model caused by a constriction of the blood vessels which was biologically implausible, and therefore the neurotransmitter hypothesis was rejected too. Instead, a new model was constructed which included elements from both the previous hypotheses. In this new model, the initial dip is caused by increased oxygen metabolism during stimulation, the post-stimulus peak is initiated by a feedforward chain of intracellular signaling triggered by glutamate released from the presynaptic neuron, and the post-peak undershoot is caused by vascular overcompensation as the CBF returns to normal (see Figure 8 in Paper I). In Paper IV, this model was extended with a GABA signaling mechanism which allowed the model to simulate a negative BOLD response caused by neuronal inhibition (a full representation of the model is shown in Figure 5.2).

\subsection{Aim 2: Explain the Positive and the Negative BOLD Response Through Activation and Inhi- bition}

It is important that the models can describe the BOLD response not just in a theoretical sense, but also in comparison to real data. Therefore, the models were fitted to measured data from healthy adults. In Paper I, curves from the posi- 


\section{Model representation of the neurovascular signalling pathway}

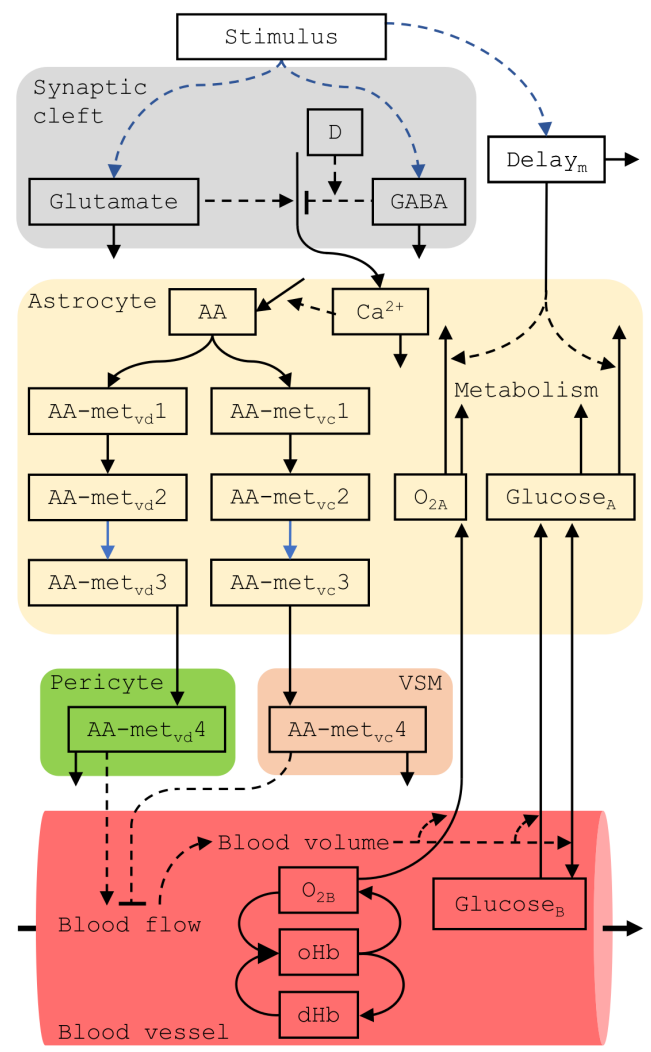

Figure 5.2: Model representation of the neurovascular coupling with both activation and inhibition, as well as the diazepam effect on the GABA receptors. Adapted from Paper IV. Each box represents a state, while non-boxes represent variables. Mass-action kinetics are shown with fully drawn arrows, while enzymereactions or non-mass action kinetics are shown in dashed lines. The output signal is formulated as BOLDsignal $=e^{k_{y}[d H b]}$. The kinetic rate parameters of the highlighted reactions (blue arrows) changes between simulation of a positive and a negative response, while non-highlighted reactions (black arrows) remain unchanged. Stimulus = input signal. $\mathrm{oHb}$ and $\mathrm{dHb}$ are oxyhemoglobin and deoxyhemoglobin, respectively and, $\mathrm{D}=$ diazepam (if present). Subscript $A$ and $B$ indicate astrocytic and blood compartment, respectively 
tive BOLD response were extracted from the visual cortex during an event-related visual task. The model parameters were estimated by fitting the model to the measured BOLD response from an event-related visual task, namely observing a white dot on a black background as it appeared for 0.5 second. In the experimental condition used for fitting the model, at least 18 seconds passed before the next stimulus, and thus the entire BOLD response could be measured and the mean curve for all individuals was calculated. This type of stimulus was called the primary stimulus and was used in two experiments. None of the measured BOLD responses showed an initial dip, but both had a post-stimulus peak and a post-peak undershoot. The final model from Paper I could be fitted to the primary stimulus from both datasets with the lowest cost being $7.2\left(\chi^{2}\right.$ threshold $\left.=49.8\right)$, Figure 5.3A. In Paper IV, both the positive BOLD response of the visual cortex and the negative BOLD response of the posterior cingulate cortex were measured in an event-related visual-motor task and the model could fit both with a cost of $27\left(\chi^{2}\right.$ threshold $\left.=72\right)$. The fit to the negative BOLD response is shown in Figure 5.3B. In a more complicated working-memory task in the same paper, the positive BOLD response was measured in the dorsolateral prefrontal cortex and the negative BOLD response of the posterior cingulate cortex. The model could fit the BOLD responses from one sequence of task order with a cost of 24 ( $\chi^{2}$ threshold $=106$ ) and it could qualitatively predict the BOLD responses of a different task order (see Figure 5 in Paper IV). The model from Paper I was also validated by successfully predicting the BOLD responses to variations in intensity and time before the next stimulus (see Figure 5.3 C and D in this section, and Figure 9 and 10 in Paper I)).

\subsection{Aim 3: Potential Biomarkers}

One of the advantages with mechanistic modelling is that observable model properties can be used as biomarkers. Paper II identifies two observable properties in a model simulating only the positive BOLD response. Both these observable properties are closely connected to the vascular control branches of the intracellular signaling chain of the model. One of the properties, $\Delta b f c$, which measured the balance between the vasoconstricting action and the vasodilatory action of the model, is more closely investigated. If the $\Delta b f c=0$ there is no change in the balance between the vasoactive substances cause by a stimulus. Using this model property in a PPL analysis where the cost of the model is measured in the fit to the fMRI signal, it is possible to differentiate between activity and noise for an event-related stimulus in both simulated data and measured data, see Figure 5.4. The simulated data was then used to take the analysis one step further. Once again the models ability to predict was used, and in a simulated dataset with a block de- 

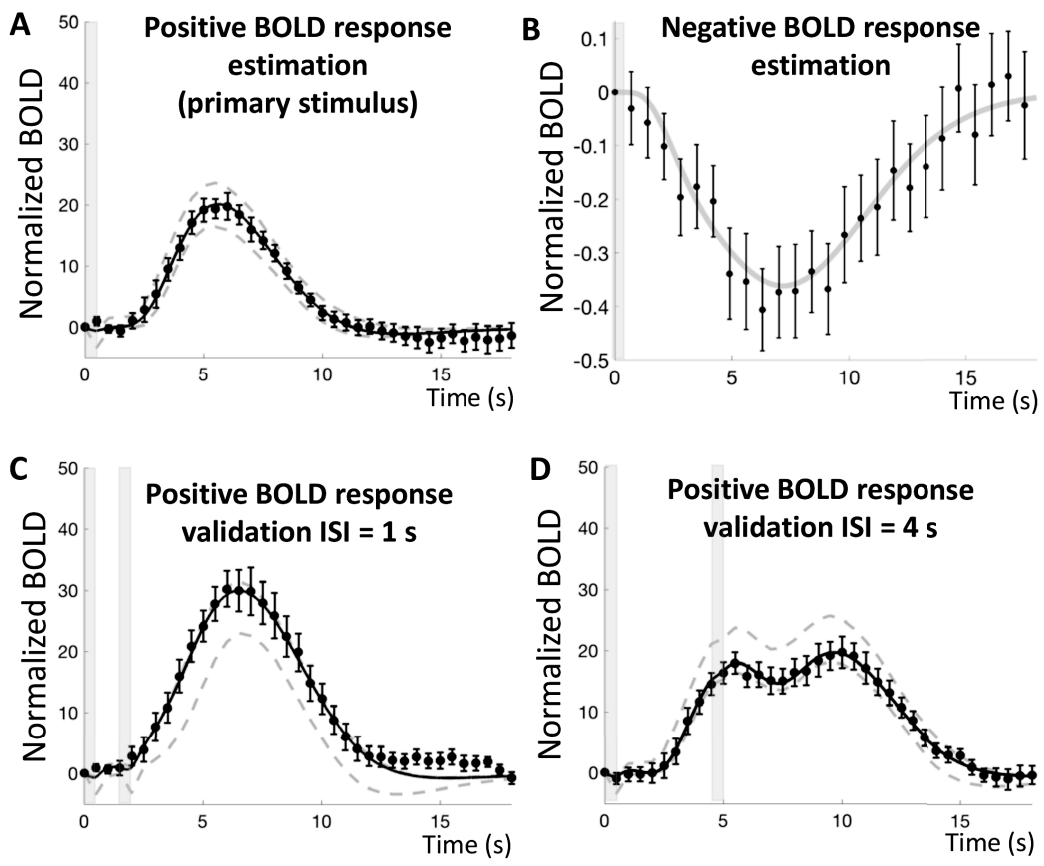

Figure 5.3: Measured and simulated BOLD responses, shaded grey area shows stimulus duration. Measured mean and SE (errorbars) and model simulation (black line) with model uncertainty (grey dashed line). A. Model fit to positive BOLD response to primary stimulus $(0.5 \mathrm{~s})$ in the visual cortex. B. Model fit to negative BOLD response in the posterior cingulate cortex during a visual-motor task. C. Prediction of the BOLD response to two stimuli with $I S I=1 \mathrm{~s}$ based on parameter values estimated in $A$ and validation data. D. Prediction of the BOLD response to two stimuli with $I S I=4 \mathrm{~s}$ based on parameter values estimated in $\mathrm{A}$ and validation data. 
sign where many short stimuli were given with short ISI, the previously described PPL analysis could still be used, as the model property $\Delta b f c$ was predicted for the whole duration of the BOLD response for each step. Using simulated data where the stimulus strength was changed, it was also shown that in the PPL analysis, the model could separate between different input strength down to $60 \%$ off the original input.
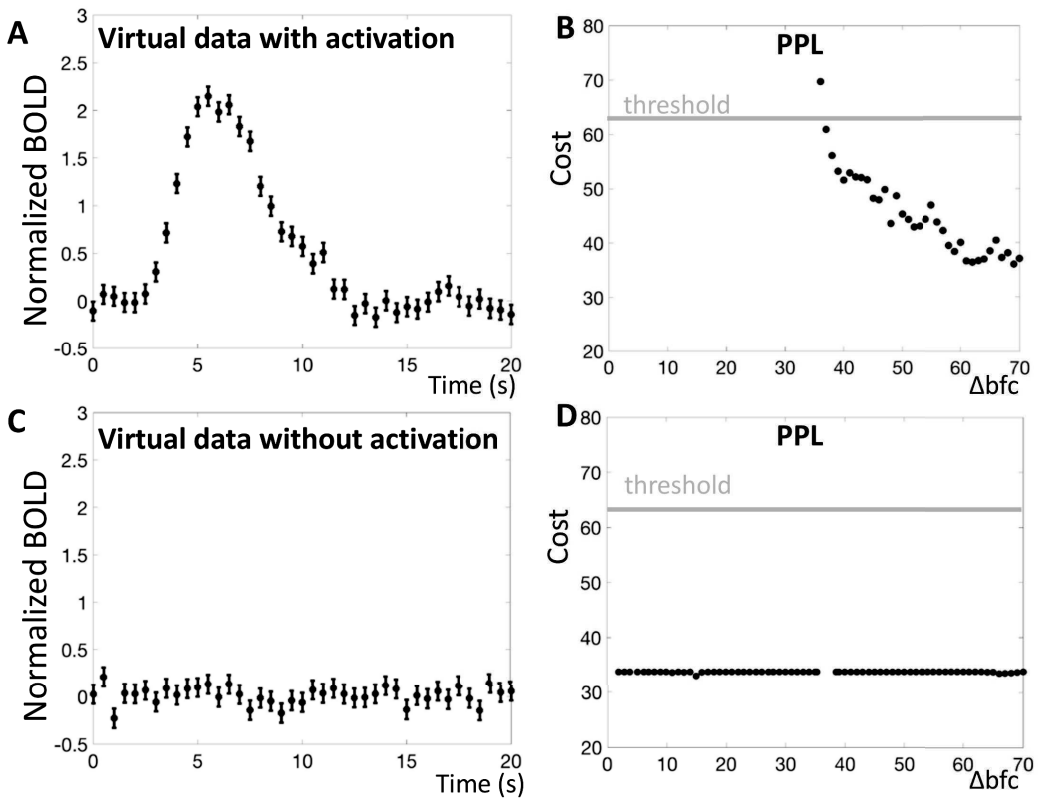

Figure 5.4: A. Simulated BOLD response with activation that the model is fitted to. B PPL graph showing that the semi-obeservable model property $\Delta b f c$ have a lower limit when the model is fitted to data with activity. C. Simulated noise. D. PPL graph showing that the semi-observable model property $\Delta b f c$ does not have a lower limit when being fitted to noise. Comparison of $B$ and $D$ shows that this type if analysis can identify activity.

\subsection{Aim 4: Pharmaceutical Modulation of GABA}

In Paper III and Paper IV, the effect of the positive allosteric modulator of GABA (diazepam) on the BOLD response was investigated. The main result is shown in Figure 5.5. In the anterior mid cingulate cortex, the BOLD response turned from 
positive to negative when the test subjects had a diazepam plasma concentration of more than $0.01 \mathrm{mg} / \mathrm{L}$. The GABA-extended model was used to simulate this effect of diazepam by adding a mechanism of GABA-receptor sensitization. The model could quantiatively predict the positive BOLD response in the measured data under placebo conditions $\left(\operatorname{cost}=30, \chi^{2}\right.$ threshold $\left.=106\right)$ and qualitatively describe the negative BOLD response under diazepam conditions $(\operatorname{cost}=243)$. The sensitization of the GABA-receptors made it possible for the model to turn the BOLD response negative without changing the amount of glutamate or GABA released. 


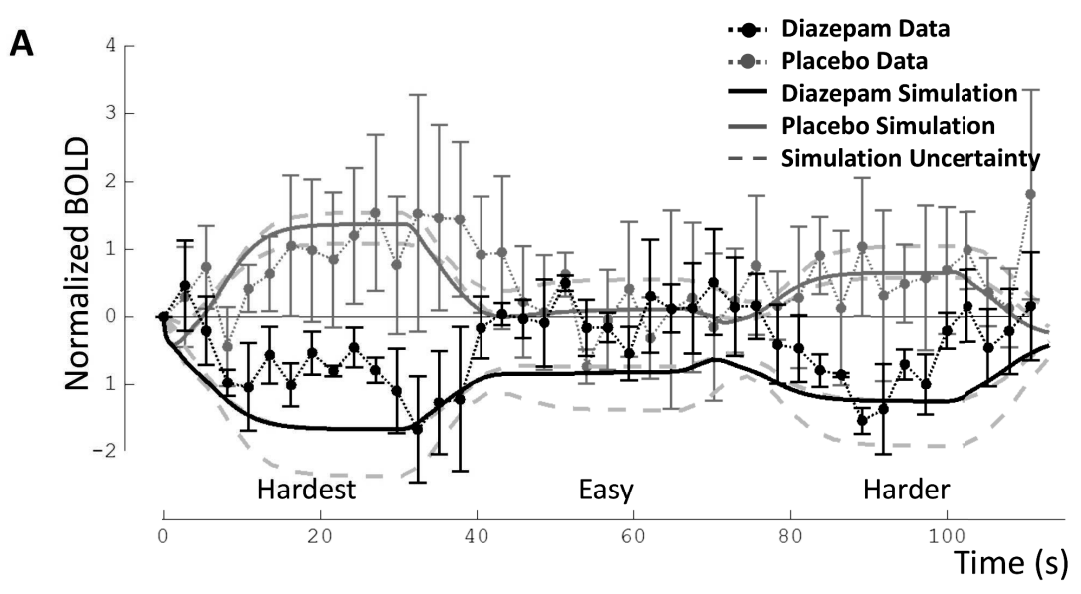

B

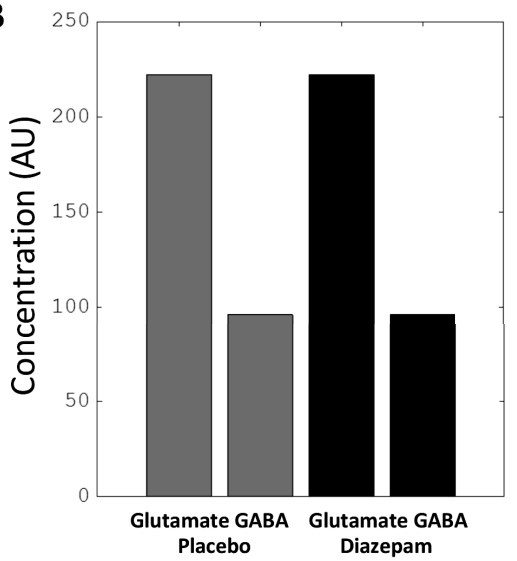

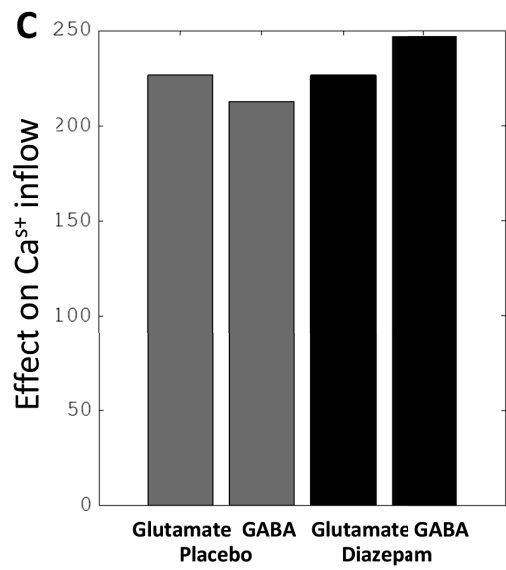

Figure 5.5: The effect of diazepam on the BOLD response. A. Model simulations of the BOLD response with experimental data from the same ROI during the working-memory task. The BOLD response is positive during the placebo condition (grey), but when influenced by diazepam, the BOLD response turns negative (black). B and C. Model mechanism for diazepam effect. The relative levels of glutamate and GABA remain unaffected by the administration of diazepam in the model simulations (B). However, diazepam increases the effect of GABA on the $\mathrm{Ca}^{2+}$ inflow, while the effect of glutamate remains the same (C). $\mathrm{AU}=$ arbitrary units and $A \cup C=$ area under curve of state time series. Easy - Hard - Hardest indicate task level of difficulty. 
"What makes one step a giant leap is all the steps before."

Leslie Fish, Toast to unsung heroes 


\section{Chapter 6}

\section{Discussion}

\subsection{Systems biology for hypothesis testing}

The three major strengths of a systems biology framework for iterative hypothesis testing are: 1) its ability to falsify hypotheses, 2) its strong basis in the biological mechanisms, and 3) its holistic perspective of the system.

Falsification of hypotheses have been an important part of the scientific method since Karl Popper introduced it in 1932, and the strongest claims that can be done using the mechanistic model analysis are rejections of hypotheses. An example of this is shown in Paper I, where the metabolic hypothesis is rejected. However, the more tests the model can pass, the more reliable the hypothesis becomes.

In section 3.3, I discussed the advantages of systems biology in an iterative study design. Using this systems biology framework, we have built a series of mechanistic models of the neurovascular coupling. They are to our knowledge the first mechanistic models based on the neurotransmitter hypothesis. The models have been used throughout this thesis to test hypotheses and design experiments. The neurotransmitter hypothesis have been modelled and repeatedly tested and validated for several aspects of the BOLD response. That the models in $\mathrm{Pa}$ per I and IV pass several tests, such as simulating the classical and the negative BOLD response, fit to measured data, predict validation data, and simulating the BOLD response change to diazepam, shows that the neurotransmitter hypothesis is a plausible and reliable explanation for the generation of the BOLD response. Mechanistic modelling can therefore be seen as a valuable complement to experimental work, not only to plan new experiment effectively but also to test assumptions and to find new and perhaps non-intuitive, explanations for complex data.

Thanks to animal experiments, we know a lot about the underlying mechanisms of the BOLD response which generates the fMRI signal. However, this knowledge is not typically used in the analysis of the fMRI signal. If information 
about the neuronal activity can still be found in the measured signal, new methods are needed to extract it. Systems biology holds the possibility of bringing two pieces of this puzzle together: the information rich fMRI signal and the extensive mechanistic knowledge.

\subsection{Potential applications in network studies}

The future of brain research lies in the study of system properties, but network organization and multiple functionality of the brain provides a challenging system to understand. "What does the neuronal excitation look like during stimulation?", "In which order are brain regions activated?" and "How does the signal change from stimulus to response?" are some of the most interesting questions being posed. The study of these types of questions in humans requires a measuring system that is as safe as possible for the test subjects, yet delivers useful information of the brain function. fMRI is such a measuring system. It is noninvasive, can be used multiple times in the same individual and can measure the whole brain with a comparatively high temporal resolution and an excellent spatial resolution. Yet, despite all these advantages, fMRI fails to answer the questions posed above because of the loss of information between the neuronal activity and the measured fMRI signal. In order to be able to use fMRI data to understand interactions, we must first understand the neurovascular coupling. Mechanistic models can both use the theoretical knowledge that we have of the system, and include data from other measuring techniques, such as EEG, optogenetics, and ASL. Observable properties brings a new measurement based on the mechanisms of the neurovascular coupling rather than an expectation of what the data should look like. Using PPL analysis on the unique time series of each voxel in the fMRI brain image, a new type of activation map can be constructed, which can potentially help us trace the signal through the network and answer the questions posed in the beginning of this section.

\subsection{Biomarkers and personalized medicine}

The use for mechanistic models in the clinic is growing steadily and model-based biomarkers and personalized medicine are two possible applications for such models.

Model-based biomarkers can be effective tools for diagnosing diseases and disorders that we do not have tests for today. One example is the sleep disorder Klein-Levines syndrome (KLS), which there is no way to test for today. Instead, KLS is diagnosed by excluding, among other things, depression and narcolepsy. 
An observable model property that displays a different span of values in patients compared to healthy controls could provide such a test. If such a model property is also physiologically interpretable, it can give important clues to the cause of the disease or disorder, pointing out the best direction to take in the next turn of the iterative study design.

Personalized medicine is a field that health care companies invest more and more resources in. Mechanistic models with parameters estimated to each individual have the potential to tailor treatments e.g. by calculating which drug and which dose of the drug would be most effective for that individual. Using the diazepam study from Papers III and IV, one could e.g. fit the model to an individual and then calculate what dose of diazepam is needed to give the desired inhibition of the cingulate cortex. Of course, first we would need to confirm that inhibition of the cingulate cortex is desirable and that the model is reliably predicting the response in different individuals for a different dose. However, the reasoning can be applied to other types of psychopharmaca and other parts of the brain. This would increase the quality of the treatment by speeding up the process of figuring out which drug that is effective for the individual and to avoid excessive use leading to unwanted side-effects. 


\section{Conclusion}

In this thesis I have used systems biology to bring the rich knowledge of the mechanisms of the neurovascular coupling into the analysis of the fMRI-signal. We have built a model of the neurovascular coupling based on the neurotransmitter hypothesis. This model is useful, as it can both explain and predict measured data for the positive and the negative BOLD response, separate between noise and data containing activity, and explain the action of diazepam on the GABA receptors leading to inhibition of the brain activity. The mechanistic model of the neurovascular coupling presented in this thesis gives us access to information in the fMRI data we did not have before, and this information have potential uses in both brain research and the clinic. 


\section{Acknowledgements}

No one can do this type of project on their own, and I owe thanks to many who have helped me. First of all, my supervisors:

Maria Engström, I am so happy that I have had you as my main supervisor. In a rushing, hurrying world, you dare to really stop and think things through to make sure we get to the bottom of it and see the true values. You have been a steady rock throughout this project.

Gunnar Cedersund, I am grateful that you have shared with me your knowledge about the frustrations and joys of working with models and trying to make them not only function, but also to fit the real world. My own world is bigger for it. You have also shown me the value of a big network and of advertising yourself, and I try to take those lessons to heart and apply them.

Fredrik Elinder, I have really appreciated having you at our monthly meetings. What I admire most about you is you eagle eye view. You see the bigger picture at all times, but at the same time you can pinpoint exactly the detail which makes the whole argument fall. I also appreciate and admire your willingness to sacrifice speed for quality: this seems to me a necessary tactic for high quality science, but one which few people dare to implement.

Susanna Walter, thank you for your constant enthusiasm and cheer even though our interactions have been brief. I am glad we finally have an article together!

There are plenty of others to thank as well:

Sebastian Sten for taking such good care of my little baby model and now taking over to raise it to reach its full potential (no pressure!).

Rikard Johansson for always giving generously of your time and explaining things to me, without ever making me feel stupid about it.

Elin Nyman for showing me the importance of caring fiercely about things - and of choosing when not to care.

Natasha Drissi Morales for all the discussions and for educating me in all things important, from the compatibility of EEG and fMRI to which animes I really ought to watch.

Helene von Ettinger Veenstra for tutoring me and helping me beat SPM into 
submission. For the future!

The other PhD students and all the other people in the fika room: Thobias, Anette, Mikael, Jonatan, Johannes, Patrik, William, Sofie, Markus, Anna, Roz and all the rest for support, commiseration and (sometimes literally) fantastic discussions during fika.

Researchers, technicians and nurses at CMIV. Especially Suzanne who helped me sort out a great deal of things, and Gerry, Björn and Dennis who were always there to wrestle the all too unwieldily computer system into submission for me.

Daniel Jönsson for the visualization.

All my minions: Theresia, Philip, Zaheer, Theo, Alex, Felix, Lisa, Bea, Johan and the students of the systems biology course, the D-program and the medical programs.

Elin Nyman, Helene van Ettinger Veenstra, Isa Lindgren, Malin Björnsdotter Åberg and Tove Bjerg for decisively claiming your space and being such awesome role models.

Jenni (the winner!) and the other "name my thesis" contestants, and all of you who proofread and commented my thesis, despite the short deadlines.

Uno Wennergren and Jordi Altimiras for paving the way for my interest in modelling and for helping me through the mess that the university made of my undergraduate program. And even earlier, Tore Mellgard who had such curious attitude to natural science and who convinced me that mathematics are not so bad after all.

Alexander Arvidsson for being such an excellent whetstone for me to hone my mind on.

Johanna Karlsson for being encouraging and curious and scientific and sometimes random when I though I knew exactly where I had you. Thank you for all the in depth discussions of presentation technique that helped further my interest in science communication.

Katarina Bengtsson for you crafting evenings (such a stress relief!) and you support in matters large and small.

My family: my brother Karl Lundengård who steps up and just fixes everything when asked. My mother Gunilla Sundström for encouraging me to walk my way even though you could not understand why I chose to research the most boring curve in the world. My father Stefan Lundengård, I am sorry that you could not attend my thesis defense, since it would have given you such pride.

Finally, all of you who came by with lunch and cheer and occasionally did my dishes for me. Especially Emma Lundquist for being such a patient and inquisitive listener, and my personal hustomte Dan Grönlund for ceaselessly taking care of me and for questioning everything. Especially when you don't have a clue how it works. Because that makes me see the things that I don't know even though I thought I did. 


\section{References}

[1] S Vossel, JJ Geng, and GR Fink. Dorsal and ventral attention systems: Distinct neural circuits but collaborative roles. The Neuroscientist, 20(2):150159, 2014.

[2] D Sridharan, D J Levitin, and V Menon. A critical role for the right frontoinsular cortex in switching between central-executive and default-mode networks. Proceedings of the National Academy of Sciences of the United States of America, 105(34):12569-12574, 2008.

[3] KK Kwong, JW Belliveau, DA Chesler, IE Goldberg, RM Weisskoff, BP Poncelet, DN Kennedy, BE Hoppel, MS Cohen, R Turner, H-M Cheng, TJ Brady, and BR Rosen. Dynamic magnetic resonance imaging of human brain activity during primary sensory stimulation. Proc. Natl. Acad. Sci. U. S. A., 89(12):5675-9, 1992.

[4] E Klipp, W Liebermeister, and C Wierling. Systems Biology: A Textbook. Wiley-Blackwell, 2008.

[5] JH Breasted, New-York Historical Society, A Stanton, and MD Friedberg. The edwin smith surgical papyrus. Rare Book Collection of Rush University Medical Center at the University of Chicago, 1930.

[6] MW Shelley. Frankenstein, Or, The Modern Prometheus. Lackington, Hughes, Harding, Mavor \& Jones, 1818.

[7] Nobel Media AB. The nobel prize in physiology or medicine 1906. Nobelprize.org., Read 22 Oct 2017.

[8] Nobel Lectures, Physiology or Medicine 1901-1921. Elsevier Publishing Company, 1967.

[9] AL Hodgkin and AF Huxley. A quantitative description of membrane current and its application to conduction and excitation in nerve. J Physiol, 117(4):500-44, 1952. 
[10] CS Roy and JG Brown. The blood pressure and its variation in the arterioles, capillaries and smaller veins. J. Physiol, 2:323-359, 1879.

[11] CS Roy and CS Sherrington. On the regulation of the blood supply of the brain. J. Physiol, 11:85-108, 1890.

[12] L Pauling and CD Coryell. The magnetic properties and structure of hemoglobin, oxyhemoglobin and carbonmonoxyhemoglobin. Proc. Natl. Acad. Sci. U. S. A., 22(4):210-216, 1936.

[13] RV Damadian, M Goldsmith, and L Minkoff. Nmr in cancer: Xvi. fonar image of the live human body. Physiol. Chem. Phys, 9(1):97-100, 1977.

[14] S Ogawa, TM Lee, AR Kay, and Tank DW. Brain magnetic resonance imaging with contrast dependent on blood oxygenation. Proc. Natl. Acad. Sci., 87:9867-9872, 1990.

[15] JW Belliveau, KK Kwong, DN Kennedy, JR Baker, CE Stern, R Benson, DA Chesler, RM Weisskoff, MS Cohen, RBH Tootell, PT Fox, TJ Brady, and Rosen BR. Magnetic resonance imaging mapping of brain function: Human visual cortex. Invest Radiol, 27:59-65, 1992.

[16] J Stelzer, G Lohmann, K Mueller, T Buschmann, and R Turner. Deficient approaches to human neuroimaging. Front. Hum. Neurosci., 8:462, 2014.

[17] SG Kim and S Ogawa. Biophysical and physiological origins of blood oxygenation level-dependent fmri signals. J. Cerb. Blood Flow Metab., 32:1188-1206, 2012.

[18] X Hu, TH Le, and K Ugurbil. Evaluation of the early response in fMRI in individual subjects using short stimulus duration. Magn Res Med, 37:877884, 1997.

[19] P Fransson, G Krüger, K-D Merboldt, and J Frahm. Temporal characteristics of oxygneation-sensitive MRI responses to visual activation in humans. Magn. Res. Med., 39:912-919, 1998.

[20] U Lindauer, G Royl, C Leithner, M Ku'uhl, L Gold, J Gethmann, M KohlBareis, A Villringer, and U Dirnagl. No evidence for early decrease in blood oxygenation in rat whisker cortex in response to functional activation. $\mathrm{Neu}$ roImage, 13:986 -999, 2001. 
[21] RD Hoge, J Atkinson, B Gill, GR Crelier, S Marrett, and GB Pike. Investigation of bold signal dependence on cerebral blood flow and oxygen consumption: the deoxyhemoglobin dilution model. Magn. Reson. Med. 42, 42:849-863, 1999a.

[22] T Jin and SG Kim. Cortical layer-dependent dynamic blood oxygenation, cerebral blood flow and cerebral blood volume responses during visual stimulation. NeuroImage, 43:1-9, 2008.

[23] JJ Chen and GB Pike. Origins of the BOLD post-stimulus undershoot. NeuroImage, 46:559-568, 2009.

[24] JB Mandeville, JJ A Marota, BE Kosofsky, JR Keltner, R Weissleder, BR Rosen, and RM Weisskoff. Dynamic functional imaging of relative cerebral blood volume during rat forepaw stimulation. Magn. Reson. Med. 39, 39:615-624, 1998.

[25] KJ Mullinger, SD Mayhew, AP Bagshaw, R Bowtell, and ST Francis. Poststimulus undershoots in cerebral blood flow and BOLD fMRI responses are modulated by poststimulus neuronal activity. Proc. Natl. Acad. Sci., 110:13636-13641, 2013.

[26] Y Nir, L Fisch, R Mukamel, H Gelbard-Sagiv, A Arieli, Fried I, and $\mathrm{R}$ Malach. Coupling between neuronal firing rate, gamma lfp, and bold fmri is related to interneuronal correlations. Curr Biol., 17(15):1275-85, 2007.

[27] I Kahn, U Knoblich, M Desai, J Bernstein, AM Graybiel, ES Boyden, RL Buckner, and CI Moore. Optogenetic drive of neocortical pyramidal neurons generates fmri signals that are correlated with spiking activity. Brain Res., 1511(33-45), 2013.

[28] NK Logothetis, J Pauls, M Augath, T Trinath, and A Oeltermann. Neurophysiological investigation of the basis of the fmri signal. Nature, 412(1507), 2001.

[29] PT Fox and ME Raichle. Focal physiological uncoupling of cerebral blood flow and oxidative metabolism during somatosensory stimulation in human subjects. Proc. Natl. Acad. Sci., 83:1140-1144, 1986.

[30] RB Buxton, EC Wong, and LR Frank. Dynamics of blood flow and oxygenation changes during brain activation: the balloon model. Magn Res Med, 39:855-864, 1998. 
[31] KJ Friston, A Mechelli, R Turner, and CJ Price. Nonlinear responses in fMRI: Balloon model, Volterra kernels, and other hemodynamics. NeuroImage, 12:473-481, 2000.

[32] A Attwell, AM Buchan, S Charpak, M Lauritzen, BA MacVicar, and EA Newman. Glial and neuronal control of brain blood flow. Nature, 468:232-243, 2010.

[33] A Mishra, JP Reynolds, Y Chen, AV Gourine, DA Rusakov, and D Attwell. Astrocytes mediate neurovascular signaling to capillary pericytes but not to arterioles. Nat. Neurosci., 19(12):1619-1627, 2016.

[34] EMC Hillman. Coupling mechanism and significance of the bold signal: a status report. Annu. Rev. Neurosci, 37:161-181, 2014.

[35] E.A. Newman. Glial cell regulation of neuronal activity and blood flow in the retina by release of gliotransmitters. Philos. Trans. R. Soc. Lond. B. Biol. Sci., 370(1672), 2015.

[36] GL Shulman, JA Fiez, M Corbetta, RL Buckner, FM Miezin, ME Raichle, and SE Petersen. Common blood flow changes across visual tasks: ii. decreases in cerebral cortex. J. Cogn. Neurosci., 9(5):648-663, 1997.

[37] ME Raichle, AM MacLeod, AZ Snyder, WJ Powers, DA Gusnard, and GL Shulman. A default mode of brain function. Proc. Natl. Acad. Sci. U. S. A., 98(2):676-682, 2001.

[38] H Lu, Q Zou, H Gu, ME Raichle, EA Stein, and Y Yang. Rat brains also have a default mode network. Proc. Natl. Acad. Sci. U. S. A., 109(10):3979-3984, 2012 .

[39] A Devor, I Ulbert, AK Dunn, SN Narayanan, SR Jones, ML Andermann, DA Boas, and AM Dale. Coupling of the cortical hemodynamic response to cortical and thalamic neuronal activity. Proc. Natl. Acad. Sci. U. S. A., 102(10):3822-3827, 2005.

[40] N Harel, S Lee, T Nagaoka, D Kim, and S Kim. Origin of negative blood oxygenation level dependent fmri signals. J. Cereb. Blood Flow. Metab. Off. J. Int. Soc. Cereb. Blood Flow. Metab., 22(8):908-917, 2002.

[41] SS Kannurpatti and BB Biswal. Negative functional response to sensory stimulation and its origins. J. Cereb. Blood Flow. Metab. Off. J. Int. Soc. Cereb. Blood Flow. Metab., 24(6):703-712, 2004. 
[42] K Schafer, F Blankenburg, R Kupers, JM Gruner, I Law, M Lauritzen, and HBW Larsson. Negative bold signal changes in ipsilateral primary somatosensory cortex are associated with perfusion decreases and behavioral evidence for functional inhibition. NeuroImage, 59(4):3119-3127, 2012.

[43] CM Klingner, C Hasler, S Brodoehl, and OW Witte. Dependence of the negative bold response on somatosensory stimulus intensity. Neuroimage, 53(1):189-195, 2010.

[44] U Schridde, M Khubchandani, JE Motelow, BG Sanganahalli, F Hyder, and $\mathrm{H}$ Blumenfeld. Negative bold with large increases in neuronal activity. Cereb. Cortex, 18(8):1814-1827, 2008.

[45] T Nagaoka, F Zhao, P Wang, N Harel, RP Kennan, S Ogawa, and S-G Kim. Increases in oxygen consumption without cerebral blood volume change during visual stimulation under hypotension condition. J. Cereb. Blood Flow Metab., 26(8):1043-1051, 2006.

[46] A Shmuel, E Yacoub, J Pfeuffer, P de Moortele, G Adriany, X Hu, and K Ugurbil. Sustained negative bold, blood flow and oxygen consumption response and its coupling to the positive response in the human brain. Neuron, 36(6):1195-1210, 2002.

[47] A Shmuel, M Augath, A Oeltermann, and NK Logothetis. Negative functional mri response correlates with decreases in neuronal activity in monkey visual area v1. Nat. Neurosci., 9(4):569-577, 2006.

[48] B Stefanovic, JM Warnking, and GB Pike. Hemodynamic and metabolic responses to neuronal inhibition. NeuroImage, 22(2):771-778, 2004.

[49] K.J. Mullinger, S.D. Mayhew, A.P. Bagshaw, R. Bowtell, and S.T. Francis. Evidence that the negative bold response is neuronal in origin: a simultaneous eeg- bold-cbf study in humans. NeuroImage, 94:263-274, 2014.

[50] L Boorman, AJ Kennerley, D Johnston, M Jones, Y Zheng, P Redgrave, and J Berwick. Negative blood oxygen level dependence in the rat: a model for investigating the role of suppression in neurovascular coupling. J. Neurosci., 30(12):4285-4294, 2010.

[51] WJ Bentley, JM Li, AZ Snyder, ME Raichle, and LH Snyder. Oxygen level and lfp in task-positive and task-negative areas: bridging bold fmri and electrophysiology. Cereb. Cortex, 26(1):346-357, 2016. 
[52] A Devor, EMC Hillman, P Tian, C Waeber, IC Teng, L Ruvinskaya, MH Shalinsky, H Zhu, RH Haslinger, SN Narayanan, I Ulbert, AK Dunn, EH Lo, BR Rosen, AM Dale, D Kleinfeld, and DA Boas. Stimulus- induced changes in blood flow and 2-deoxyglucose uptake dissociate in ipsilateral somatosensory cortex. J. Neurosci., 28(53):14347-14357, 2008.

[53] G Buzsáki, K Kaila, and M Raichle. Inhibition and brain work. Neuron, 56(5):771-783, 2007.

[54] SR Jilka, G Scott, T Ham, A Pickering, V Bonnelle, RM Braga, R Leech, and DJ Sharp. Damage to the salience network and interactions with the default mode network. The Journal of Neuroscience, 34(33):10798-10807, 2014.

[55] W Sieghart. Structure and pharmacology of gamma-aminobutyric acida receptor subtypes. Pharmacol. Rev., 47(2):181-234, 1995.

[56] C Braestrup and RF Squires. Specific benzodiazepine receptors in rat brain characterized by high-affinity (3h)diazepam binding. Proc. Natl. Acad. Sci. U. S. A., 74(9):3805-3809, 1977.

[57] RE Twyman, CJ Rogers, and RL Macdonald. Differential regulation of gamma-aminobutyric acid receptor channels by diazepam and phenobarbital. Ann. Neurol., 25(3):213-220, 1989.

[58] SA Huettel, AW Song, and G McCarthy. Functional Magnetic Resonance Imaging. Sinauer, 2nd edition, 2009.

[59] JA Mumford, RA Poldrack, and TE Nichols. Handbook of Functional MRI Data Analysis. Cambridge University Press, 2011.

[60] RL Buckner, PA Bandettini, KM O’Craven, RL Savoy, SE Petersen, ME Raichle, and BR Rosen. Detection of cortical activation during averaged single trials of a cognitive task using functional magnetic resonance imaging. Proc. Natl. Acad. Sci. U. S. A., 93(25):14878-14883, 1996.

[61] GM Boynton, SA Engel, GH Glover, and DJ Heeger. Linear systems analysis of functional magnetic resonance imaging in human v1. J Neurosci., 16(13):4207-21, 1996.

[62] AL Vazquez and DC Noll. Nonlinear aspects of the bold response in functional mri. NeuroImage, 7(2):108-18, 1998. 
[63] D Caplan, S Vijayan, G Kuperberg, C West, G Waters, D Greve, and AM Dale. Vascular responses to syntactic processing: event-related fmri study of relative clauses. Hum Brain Mapp., 15(1):26-38, 2002.

[64] AP Holmes and KJ Friston. Generalizability, random effects, and population inference. Neuroimage and Proceedings of the Fourth International Conference on Functional Mapping of the Human Brain, June 7-12, 1998, Montreal, Canada., 7:754, 1999.

[65] MW Woolrich, TE Behrens, CF Beckmann, M Jenkinson, and SM Smith. Multilevel linear modelling for fmri group analysis using bayesian inference. NeuroImage, 21(4):1732-47, 2004.

[66] CF Beckmann and SM Smith. Probabilistic independent component analysis for functional magnetic resonance imaging. IEEE Trans Med Imaging., 23(2):137-52, 2004.

[67] AR McIntosh and B Mišić. Multivariate statistical analyses for neuroimaging data. Annu Rev Psychol., 64(499-525), 2013.

[68] VD Calhoun and T Adali. Unmixing fmri with independent component analysis. IEEE Engineering in Medicine and Biology Society, 25(2), 2006.

[69] KJ Friston, L Harrison, and W Penny. Dynamic causal modelling. NeuroImage, 19(4):1273-302, 2003.

[70] ST Witt, M Warntjes, and M Engström. Increased fmri sensitivity at equal data burden using averaged shifted echo acquisition. Front Neurosci., 10:544, 2016.

[71] H Kitano. Computational systems biology. Nature, 420:206-210, 2002.

[72] H Schmidt and M Jirstrand. Systems biology toolbox for matlab: A computational platform for research in systems biology. Bioinformatics, 22:514$515,2015$.

[73] G Cedersund. Conclusions via unique predictions obtained despite unidentifiability - new definitions and a general method. FEBS J, 279:3513-3527, 2012 .

[74] G Cedersund and J Roll. Systems biology: model based evaluation and comparison of potential explanations for given biological data. FEBS J, 276:903-922, 2009. 
[75] G Bluman and S Anco. Symmetry and Integration Methods for Differential Equations. Springer New York, 2008.

[76] A Raue, M Schilling, J Bachmann, A Matteson, M Schelke, D Kaschek, S Hug, C Kreutz, BD Harms, FJ Theis, U Klingmüller, and J Timmer. Lessons learned from quantitative dynamical modeling in systems biology. PLoS One, 8(9), 2013.

[77] A Raue, C Kreutz, T Maiwald, U Klingmüller, and J Timmer. Addressing parameter identifiability by model-based experimentation. IET Systems Biology, 2010.

[78] T Maiwald, J Timmer, C Kreutz, U Klingmüller, and A Raue. Addressing parameter identifiability by model-based experimentation. IET Syst. Biol., 5(2):120-30, 2011.

[79] A Raue, C Kreutz, T Maiwald, J Bachmann, M Schilling, U Klingmüller, and J Timmer. Structural and practical identifiability analysis of partially observed dynamical models by exploiting the profile likelihood. Bioinformatics, 25(15):1923-9, 2009.

[80] MF Forsgren, O Dahlqvist Leinhard, N Dahlström, G Cedersund, and $\mathrm{P}$ Lundberg. Physiologically realistic and validated mathematical liver model reveals [corrected] hepatobiliary transfer rates for gd-eob-dtpa using human dce-mri data. PLoS One, 9(4), 2014. 


\section{Papers}

The papers associated with this thesis have been removed for copyright reasons. For more details about these see:

http:// urn.kb.se/ resolve?urn=urn:nbn:se:liu:diva-142870 